\title{
A Systematic Review and Metanalyis of Soil Transmitted Helminths Infections Among Preschool and School-age Children in Ethiopia: Evaluation of Neglected Tropica Diseases (NTDS) Elimination Program by 2020
}

Legese Chelkeba ( $\square$ legese.chelkeba@gmail.com)

Jimma Universit https://orcid.org/0000-0002-0323-5011

Tsegaye Melaku

Jimma University College of Public Health and Medical Sciences

Daniel Emana

Jimma University College of Public Health and Medical Sciences

Worku Jimma

Jimma University College of Public Health and Medical Sciences

\section{Research}

Keywords: Soil transmitted helminths, preschool-age children, school-age children, Meta-analysis, Ethiopia

Posted Date: June 9th, 2020

DOI: https://doi.org/10.21203/rs.3.rs-32212/v1

License: @ (1) This work is licensed under a Creative Commons Attribution 4.0 International License. Read Full License 


\section{Abstract}

Background: Comprehensive nationwide on prevalence, geographic distribution of different species and time trends of soil-transmitted helminths (STHs) are lacking. Therefore, the aim of this study was to provide a summary and location of the available data on STHs infection among preschool and school-age children in Ethiopia.

Methods: The search were carried out in Medline via PubMed, Scopus, Science Direct, Web of Science and Google Scholar on data published between 1995 to Fabruary 2020 for studies describing rate of STHs infection among preschool and school-age in Ethiopian. We followed Patient, intervention, Comparsion and Outcome (PICO) approach to identify the studies. Endnote citation manager software version $\mathrm{X} 9$ for Windows was utilized to collect and organize search outcomes (into relevant and irrelevant studies) and for removal duplicate articles. We conducted meta-regression to understand the trends and the source of heterogeneity and pooled the prevalence using 'metaprop' command using STATA software version 14.

Results: A total of 29,311 of the 61,690 children examined during the period under review were infected with one or more species of intestinal parasites yielding an overall prevalence of $48 \%$ (95\% Cl: $43-53 \%)$. The overall pooled estimate of STHs was 33\% $(95 \% \mathrm{Cl}$ : $28-38 \%)$. The prevalence was $44 \%(95 \% \mathrm{Cl}: 31 \%-58 \%)$ in SNNPR, $34 \%((95 \% \mathrm{Cl}: 28 \%-41 \%)$ in Amhara region, $31 \%(95 \% \mathrm{Cl}: 19 \%$ $43 \%)$ in Oromia region and $10 \%(95 \% \mathrm{Cl}: 7 \%-12 \%)$ in Tigray region. Soil-transmitted helminths infection rate has been decreasing from 44\% (95\% Cl:30-57\%) pre-Mass Drug Administration (MDA) era (1997-2012) to 30\% (95\% Cl:25-34\%) post-MDA (2013-2020), although statistically not significant $(p=0.45)$. A. lumbricoides was the predominant species with a prevalence of $17 \%$.

Conclusion: Southern Nations Nationalities and Peoples Region , Amhara and Oromia regions carry the highest burden and are categorized to Moderate Risk Zones (MRZ) and therefore, requiring mass drug administration (MDA) once annually with Albendazole or Mebendazole. Prevalence of SHTs decreased after MDA compared to before MDA, but the decline was not statistically significant. A. lumbricoides was the predominant species of STHs among preschool and school-age children in Ethiopia.

\section{Background}

Soil transmitted helminths (STHs) infections have been among the most widely distributed infections in the resources limited countries. Globally, more than 4.5 billion people are at risk of infection with nearly 2 billion are infected with STHs [1, 2]. In contrast to other infectious diseases, infection due to STHs such as Ascaris lumbricoides, hookworm species and Trichuris trichiura do not usually cause significant mortality rates; instead adapted to chronic illness and extended morbidity affecting poor people [3-6]

Transmissions of the STHs are mainly by eggs or larvae that are passed with feces of infected person or hatched in soil after defecation. Adult worms residing in the gut of infected person produce thousands of eggs every day, which may contaminate environments or foods that lack adequate sanitation [7]. Additionally, climatic conditions of tropical and sub-tropical countries are suitable for the survival of STH eggs and larvae hatching and embryonation in warm temperature and adequate moisture soil [8]. Consequently, the complication of STH may cause gut blood losses, malabsorption of nutrients, loss of appetites and anemia due to loss of iron and other important protein [9]. For instance, the infections outcome on the children results in serious problems such as anemia, growth retardation, impaired cognitive developments, school absenteeism and disability adjusted life years lost $[10,11]$.

World Health Organization (WHO) has published a comprehensive road map data in 2012 to combat Neglected tropical diseases (NTDs) by 2020. Mass Drug Administration (MDA) approach was also designed to undertake $75 \%$ coverage in all of known endemic countries for STHs. The ideas of WHO was strengthen by the London declaration to control or eliminate other 10 NTDs in addition to the STHs $[12,13]$. Recently, following WHO strategic plan, Ethiopia has launched a nationwide MDA to control STHs, which targets 17 million children within the age range of 5-14 years old. Ethiopian Ministry of health and WHO started school children deworming in 2013 and 2014 about 6.8 million and 7.8 million school-age children, respectively. Even prior to the dewarming program, the minstry of health has undertaken some other measures to control poverty related diseases including STHs among the population at risk, particularily school-age children. For instance, implementation of health extension program focusing on creating awareness on latrine construction and utilization and keeping personal and environmental hygiene among the community is one priority program since 2003/2004. However, current individual reports indicated that the prevalence of STHs in Ethiopia is not declining. Evidently, a large scale study conducted in Amhara regional state showed that the prevalence of STHs was 36.4\% [14]. Another study in Jimma town showed that the prevalence of STHs among school-age children was 49.0\% [15]. A similar study also reported that the prevalence of STHs was $47 \%$ in rural community of Ethiopia [16]. Nevertheless, numerous fragmented studies have been carried out on assessing 
the prevalence of STHs among preschool and school-age children in Ethiopia, but a comprehensive nationwide data on the prevalence, geographic distribution of different species and time trends of STHs are lacking. Therefore, the aim of this study was to provide a summary on prevalence, geographical location and time trends of STHs among preschool and school-age children to measure the impact of the ongoing control and preventive measures in the country. In addition, such effort help the government and other conserned bodies to focus on specific areas of high prevalence for further preventive measures such as chemotherapy and improved sanitation practices.

\section{Methods}

This Systematic Reviews and Meta-analyses was carried out following the PRISMA guideline (Preferred Reporting Items for Systematic Reviews and Meta- Analyses)[17]. We used the PRISMA guideline for the inclusion of potentially related studies to the outcome of interest. The outcome of interest was the prevalence of soil-transmitted helminths (STHs) among preschool and schoolage children in Ethiopia.

\section{Search Strategy}

The search were carried out in Medline via PubMed, Scopus, Science Direct, Web of Science and Google Scholar using searching terms such as "intestinal helminths", "intestinal parasites", "soil transmitted helminths", "STHs", " Strongyloides stercoralis", "Ascaris lumbricoides", " Trichuris trichiura", " Hookworms", "preschool-age", "school-age", "Ethiopia”. These key terms were combined using "AND" and "OR" Boolean operators. Medical Subject Headings (MeSH terms) was used to search relevant original articles in PubMed. Searching was carried out on articles published between 1995 to Fabrauary 2020 and limited to English language and human studies. A manual search for additional relevant studies using references from retrieved articles and related systematic reviews was also performed to identify original articles we might have missed. Endnote citation manager software version X9 for Windows was utilized to collect and organize search outcomes (into relevant and irrelevant studies) and for removal duplicate articles. We followed PICO approach to identify the relevant articles:

- Population (P): School-age children

- Exposure (E): Presence of soil-transmitted helminths

- Comparison (C): Preschool-age children

- Outcome (0): Prevalence of soil transmitted helminths.

Prevalence was calculated as the number of subjects positive for STHs in the study divided by the total number of participants in a study multiplied by 100 .

\section{Inclusion And Exclusion Criteria}

We included observational studies or controlled clinical trials conducted between 1997 to Fabruary 2020 which documented the baseline prevalence or incidence of STHs and studies published in English language targeting both pre-school ( $<5$ years) and schoolage chikdren ( $\geq 5$ years). We excluded case reports, case series, studies that compared the sensitivity and specificity of different methods for diagnosis of STHs and studies not reported either prevalence or incidence as outcome of interest.

\section{Data Abstraction And Quality Assessment}

Following preliminary assessment and downloading of the abstracts by two authors, they were assessed for agreement with the inclusion criteria. Irrelevant articles (articles which were out of the scope of the study) were excluded after assessment of the abstracts unless it was unclear to classify articles into irrelevant based on abstracts, where we downloaded the full-text for further clarity. Once articles deemed to be relevant, the full-text of the articles were downloaded for further detailed review. We extracted information on name of the first author and year of publication, study design, gender, region of study, laboratory method identification of the parasites, total sample size, the number of positives for intestinal paraistes in general, number of positive for STHs in particular, and quality score for quality assessment. The Grading of Recommendation Assessment, development and Evaluation (GRADE) 
approach was used to assess the overall quality of evidence [18]. Studies were given one point each if they had probability sampling, larger sample sizes of more than 200, and repeated detection and upto four points could be assigned to each study. We regarded publications with a total score of 3-4 points to be of high quality, whereas 2 points represented moderate quality and scores of $0-1$ represented low quality.

\section{Statistical analysis}

We used forest plots to estimate pooled effect size and effect of each study with their confidence interval (Cl) to provide a visual summary of the data. A random-effects model was used in this meta-analysis because of anticipated heterogeneity. Statistical heterogeneity among studies was expressed as the Cochrane's $Q$ test and $\mathrm{I}^{2}$, where a $\mathrm{P}<0.05$ and $\mathrm{I}^{2}$ values of $0,25,50$ and $75 \%$ were considered as no, low, moderate and high heterogeneities, respectively. Because we expected geographical variation and socioeconomic contexts might differ radically across these studies, subgroup analysis based on geography of region, age children included and year of publication. In addition to visual inspection for symmetry of the plot, we also used Begg's Funnel plot and Egger's regression test for quantitative evaluation of the possibility of publication bias. Meta-regression analysis was employed to identify the source of heterogeneity using regional states, age of children, publication years and study design as covariates. All reported $\mathrm{P}$ values were 2-sided and were statistical significant if $P<0.05$.

\section{Results}

\section{Literature searches and selection}

Our initial search of electronic databases such as Medline via PubMed, Scopus, Science direct, Web of Sciences and Google scholar yielded 953 articles and 3 articles manually from which 213 records remained after removing duplications. Upon screening the articles, 123 articles were further excluded; 112 were irrelevant because they were not specifically about preschool or school-age children, 6 studies were about sensitivity and specify of diagnosis of STHs, and 5 articles were not about human. Upon further assessment for eligibility, 2 studies were excluded being review articles. Finally, 88 [6, 14, 16, 19-110] published studies between 1997 and Fabrauary 2020 fulfilling the inclusion criteria were included in the final analyses [Figure 1]. The sample size of the included studies ranged from 100 [20] to 15,455 [14]. Atotal of 61,690 children with age of $<5$ years $(n=5577)$ and $\geq 5$ years $(n=55731)$ or mix of both $(n=382)$ were recruited in the studies. Fifty two precent $(52 \%)$ of the study participants were male. Majority (83) of the studies were cross sectional. Seventy three studies were about STHs in school-age children, 13 were about preschool-age and the rest were studies involved both preschool and school-age children. Thirty five and twenty four studies used Kato-katz or incombination with other tools and formalin-ether concentartion plus direct microscopic method for screening stools, respectively. Formalin-ether concentration techinques in 19 studies, direct wet mount method in 5 studies, McMaster in 4 studies and Harada Mori (Test tube culture) technique in one study utilized as screening of stools. According to our quality assessment criteria, 43 publications were of high quality with a score of 3,11 had a score of 2 indicating moderate quality and the remaining 34 were of low quality with a score of zero or one [Table 1]. 
Table 1

Characteristics of the eligible studies on STH infections in Ethiopia

\begin{tabular}{|c|c|c|c|c|c|c|c|c|c|}
\hline $\begin{array}{l}\text { Name of first } \\
\text { author and } \\
\text { year }\end{array}$ & $\begin{array}{l}\text { Study } \\
\text { design }\end{array}$ & Population & Male & Female & Region & $\begin{array}{l}\text { Laboratory } \\
\text { method }\end{array}$ & $\begin{array}{l}\text { No. } \\
\text { sample }\end{array}$ & $\begin{array}{l}\text { Cases } \\
\text { for } \\
\text { IPls }\end{array}$ & $\begin{array}{l}\text { Quality } \\
\text { score }\end{array}$ \\
\hline $\begin{array}{l}\text { Degarege } \\
2013[[34]\end{array}$ & $\begin{array}{l}\text { Cross- } \\
\text { sectional }\end{array}$ & $\begin{array}{l}\text { School } \\
\text { children }\end{array}$ & 187 & 216 & Amhara & KATO & 403 & 255 & 2 \\
\hline $\begin{array}{l}\text { Abdi } 2017 \\
\text { [73] }\end{array}$ & $\begin{array}{l}\text { cross- } \\
\text { sectional }\end{array}$ & $\begin{array}{l}\text { School } \\
\text { children }\end{array}$ & 207 & 201 & Amhara & FEC & 408 & 282 & 3 \\
\hline $\begin{array}{l}\text { Abera } \\
2014[74]\end{array}$ & $\begin{array}{l}\text { Cross- } \\
\text { sectional }\end{array}$ & $\begin{array}{l}\text { School } \\
\text { children }\end{array}$ & 193 & 192 & Amhara & FEC & 385 & 357 & 3 \\
\hline $\begin{array}{l}\text { Amare } \\
2013[26]\end{array}$ & $\begin{array}{l}\text { Cross- } \\
\text { sectional }\end{array}$ & $\begin{array}{l}\text { School } \\
\text { children }\end{array}$ & 218 & 187 & Amhara & KTO \&FEC & 405 & 92 & 3 \\
\hline $\begin{array}{l}\text { Gelaw } \\
2013[41]\end{array}$ & $\begin{array}{l}\text { cross- } \\
\text { sectional }\end{array}$ & $\begin{array}{l}\text { School } \\
\text { children }\end{array}$ & 170 & 134 & Amhara & DWM\&FEC & 304 & 104 & 3 \\
\hline $\begin{array}{l}\text { Abossie } \\
2014[76]\end{array}$ & $\begin{array}{l}\text { Cross- } \\
\text { sectional }\end{array}$ & $\begin{array}{l}\text { School } \\
\text { children }\end{array}$ & 191 & 209 & SNNPR & DWM\&FEC & 400 & 324 & 3 \\
\hline $\begin{array}{l}\text { Mathewos } \\
2014[53]\end{array}$ & $\begin{array}{l}\text { Cross- } \\
\text { sectional }\end{array}$ & $\begin{array}{l}\text { School } \\
\text { children }\end{array}$ & 139 & 122 & Amhara & DWM\&MZN & 261 & 174 & 2 \\
\hline $\begin{array}{l}\text { Gizaw } \\
2018[108]\end{array}$ & $\begin{array}{l}\text { Cross- } \\
\text { sectional }\end{array}$ & $\begin{array}{l}\text { Preschool } \\
\text { children }\end{array}$ & 106 & 119 & Amhara & KATO & 225 & 58 & 3 \\
\hline $\begin{array}{l}\text { Yimam } \\
\text { 2016[72] }\end{array}$ & $\begin{array}{l}\text { Cross- } \\
\text { sectional }\end{array}$ & $\begin{array}{l}\text { School } \\
\text { children }\end{array}$ & 187 & 216 & Amhara & KTO\&FEC & 403 & 235 & 3 \\
\hline $\begin{array}{l}\text { Hailegebriel } \\
\text { 2017[77] }\end{array}$ & $\begin{array}{l}\text { Cross- } \\
\text { sectional }\end{array}$ & $\begin{array}{l}\text { School } \\
\text { children }\end{array}$ & 177 & 182 & Amhara & FEC & 359 & 235 & 3 \\
\hline $\begin{array}{l}\text { Alemu } \\
\text { 2018[78]] }\end{array}$ & $\begin{array}{l}\text { Cross- } \\
\text { sectional }\end{array}$ & $\begin{array}{l}\text { School } \\
\text { children }\end{array}$ & 196 & 195 & SNNPR & FCE & 391 & 182 & 2 \\
\hline $\begin{array}{l}\text { Alemu } \\
2019[80]]\end{array}$ & $\begin{array}{l}\text { Cross- } \\
\text { sectional }\end{array}$ & $\begin{array}{l}\text { School } \\
\text { children }\end{array}$ & 180 & 171 & SNNPR & DWM\&FEC & 351 & 95 & 3 \\
\hline $\begin{array}{l}\text { Mekonnen } \\
\text { 2019[109] }\end{array}$ & $\begin{array}{l}\text { Cross- } \\
\text { sectional }\end{array}$ & $\begin{array}{l}\text { Preschool } \\
\text { children }\end{array}$ & 152 & 158 & Amhara & DWM\&KATO & 310 & 58 & 3 \\
\hline $\begin{array}{l}\text { Jejaw } \\
2015[36]\end{array}$ & $\begin{array}{l}\text { Cross- } \\
\text { sectional }\end{array}$ & $\begin{array}{l}\text { School } \\
\text { children }\end{array}$ & 228 & 232 & SNNPR & DWM,FEC\&KATO & 460 & 353 & 3 \\
\hline $\begin{array}{l}\text { Alemu } \\
\text { 2016[79] }\end{array}$ & $\begin{array}{l}\text { Cross- } \\
\text { sectional }\end{array}$ & $\begin{array}{l}\text { Preschool } \\
\text { children }\end{array}$ & 183 & 218 & Amhara & KATO & 401 & 141 & 3 \\
\hline $\begin{array}{l}\text { Alemayehu } \\
2017[54]\end{array}$ & $\begin{array}{l}\text { Cross- } \\
\text { sectional }\end{array}$ & $\begin{array}{l}\text { School } \\
\text { children }\end{array}$ & 287 & 216 & SNNPR & KATO\&FEC & 503 & 363 & 3 \\
\hline $\begin{array}{l}\text { Gashaw } \\
\text { 2015[57] }\end{array}$ & $\begin{array}{l}\text { Cross- } \\
\text { sectional }\end{array}$ & $\begin{array}{l}\text { School } \\
\text { children }\end{array}$ & 255 & 295 & Amhara & KATO & 550 & 365 & 3 \\
\hline $\begin{array}{l}\text { Amor } \\
2016[82]\end{array}$ & $\begin{array}{l}\text { Cross- } \\
\text { sectional }\end{array}$ & $\begin{array}{l}\text { School } \\
\text { children }\end{array}$ & 225 & 171 & Amhara & FEC & 396 & 327 & 3 \\
\hline $\begin{array}{l}\text { Nute } \\
2018[14]\end{array}$ & $\begin{array}{l}\text { Cross- } \\
\text { sectional }\end{array}$ & $\begin{array}{l}\text { School } \\
\text { children }\end{array}$ & 7418 & 8037 & Amhara & FEC & 15455 & 5626 & 3 \\
\hline $\begin{array}{l}\text { Zemene } \\
2018[68]\end{array}$ & $\begin{array}{l}\text { Cross- } \\
\text { sectional }\end{array}$ & $\begin{array}{l}\text { Preschool } \\
\text { children }\end{array}$ & 118 & 118 & Amhara & DWM\&FEC & 247 & 43 & 1 \\
\hline $\begin{array}{l}\text { Mulatu } 2015 \\
{[24]}\end{array}$ & $\begin{array}{l}\text { Cross- } \\
\text { sectional }\end{array}$ & Preschool & 81 & 77 & SNNPR & DWM,FEC\&MZN & 158 & 224 & 3 \\
\hline
\end{tabular}

DWM $=$ direct wet mount; FEC $=$ formal-ether: KATO = kato-katz: NNNPR = Southern nations nationalities and peoples region, IPIs = intestinal parasitic infections: STHs = soil transmitted helminths: NA = not available: 


\begin{tabular}{|c|c|c|c|c|c|c|c|c|c|}
\hline $\begin{array}{l}\text { Name of first } \\
\text { author and } \\
\text { year }\end{array}$ & $\begin{array}{l}\text { Study } \\
\text { design }\end{array}$ & Population & Male & Female & Region & $\begin{array}{l}\text { Laboratory } \\
\text { method }\end{array}$ & $\begin{array}{l}\text { No. } \\
\text { sample }\end{array}$ & $\begin{array}{l}\text { Cases } \\
\text { for } \\
\text { IPls }\end{array}$ & $\begin{array}{l}\text { Quality } \\
\text { score }\end{array}$ \\
\hline $\begin{array}{l}\text { Bekana } \\
2019[84]\end{array}$ & $\begin{array}{l}\text { Cross- } \\
\text { sectional }\end{array}$ & $\begin{array}{l}\text { School } \\
\text { children }\end{array}$ & 172 & 145 & Oromia & KATO\&FEC & 317 & 130 & 3 \\
\hline Diro 2015[85] & $\begin{array}{l}\text { prospective } \\
\text { cohort }\end{array}$ & Both & 85 & 37 & Amhara & DWM,FEC\&KATO & 122 & 371 & 1 \\
\hline $\begin{array}{l}\text { Birhanu } \\
2018[86]\end{array}$ & $\begin{array}{l}\text { cross } \\
\text { sectional }\end{array}$ & $\begin{array}{l}\text { School } \\
\text { children }\end{array}$ & 194 & 228 & $\begin{array}{l}\text { Benishangul- } \\
\text { Gumuz }\end{array}$ & DWM & 422 & 138 & 1 \\
\hline $\begin{array}{l}\text { Fentie } \\
2013[22]\end{array}$ & $\begin{array}{l}\text { Cross- } \\
\text { sectional }\end{array}$ & $\begin{array}{l}\text { School } \\
\text { children }\end{array}$ & 361 & 159 & Amhara & KATO\&FEC & 520 & 134 & 3 \\
\hline $\begin{array}{l}\text { Aiemjoy } \\
\text { 2017[63] }\end{array}$ & $\begin{array}{l}\text { Cross- } \\
\text { sectional }\end{array}$ & $\begin{array}{l}\text { Preschool } \\
\text { children }\end{array}$ & NA & NA & Amhara & FEC & 212 & 354 & 2 \\
\hline $\begin{array}{l}\text { Dessalegn } \\
\text { 2014[21] }\end{array}$ & $\begin{array}{l}\text { Cross- } \\
\text { sectional }\end{array}$ & $\begin{array}{l}\text { School } \\
\text { children }\end{array}$ & 271 & 315 & Oromia & DWM\&FEC & 586 & 91 & 3 \\
\hline $\begin{array}{l}\text { Gebrehiwot } \\
2014[110]\end{array}$ & $\begin{array}{l}\text { Cross } \\
\text { sectional }\end{array}$ & $\begin{array}{l}\text { Preschool } \\
\text { children }\end{array}$ & 195 & 179 & Oromia & KATO & 374 & 1471 & 2 \\
\hline Leta 2018[87] & $\begin{array}{l}\text { Cross } \\
\text { sectional }\end{array}$ & $\begin{array}{l}\text { School } \\
\text { children }\end{array}$ & NA & NA & Amhara & KATO & 2,650 & 437 & 3 \\
\hline King 2013[37] & $\begin{array}{l}\text { cross } \\
\text { sectional }\end{array}$ & Both & 1130 & 1228 & Amhara & FEC & 2,338 & 267 & 3 \\
\hline $\begin{array}{l}\text { Mekonnen } \\
\text { 2013[6] }\end{array}$ & $\begin{array}{l}\text { Clinical } \\
\text { trial }\end{array}$ & $\begin{array}{l}\text { School } \\
\text { children }\end{array}$ & NA & NA & Oromia & KATO & 840 & 421 & 3 \\
\hline $\begin{array}{l}\text { Mahmud } \\
\text { 2015[44] }\end{array}$ & $\begin{array}{l}\text { Clinical } \\
\text { trial }\end{array}$ & $\begin{array}{l}\text { School } \\
\text { children }\end{array}$ & 152 & 217 & Tigray & DWM,FEC\&KATO & 369 & 326 & 3 \\
\hline $\begin{array}{l}\text { Mahmud } \\
\text { 2013[43] }\end{array}$ & $\begin{array}{l}\text { cross- } \\
\text { sectional }\end{array}$ & $\begin{array}{l}\text { School } \\
\text { children }\end{array}$ & 288 & 312 & Tigray & DWM,FEC\&KATO & 600 & 89 & 3 \\
\hline $\begin{array}{l}\text { Tefera } \\
2017[70]\end{array}$ & $\begin{array}{l}\text { Cross } \\
\text { sectional }\end{array}$ & $\begin{array}{l}\text { School } \\
\text { children }\end{array}$ & 282 & 433 & Oromia & McMaster & 715 & 202 & 2 \\
\hline $\begin{array}{l}\text { Tefera } \\
2015[88]\end{array}$ & $\begin{array}{l}\text { Cross } \\
\text { sectional }\end{array}$ & $\begin{array}{l}\text { School } \\
\text { children }\end{array}$ & 364 & 280 & Oromia & McMaster & 644 & 237 & 2 \\
\hline $\begin{array}{l}\text { Nguyen } \\
2012[31]\end{array}$ & $\begin{array}{l}\text { Cross } \\
\text { sectional }\end{array}$ & $\begin{array}{l}\text { School } \\
\text { children }\end{array}$ & 341 & 323 & Amhara & FEC & 664 & 129 & 3 \\
\hline $\begin{array}{l}\text { Hailu } \\
\text { 2018[111] }\end{array}$ & $\begin{array}{l}\text { Cross } \\
\text { sectional }\end{array}$ & $\begin{array}{l}\text { School } \\
\text { children }\end{array}$ & 186 & 223 & Amhara & Richie's & 409 & 263 & 2 \\
\hline $\begin{array}{l}\text { Beyene } \\
2014[28]\end{array}$ & $\begin{array}{l}\text { Cross } \\
\text { sectional }\end{array}$ & $\begin{array}{l}\text { School } \\
\text { children }\end{array}$ & 114 & 146 & Oromia & DWM\&FEC & 260 & 328 & 3 \\
\hline $\begin{array}{l}\text { Alemu } \\
2011[25]\end{array}$ & $\begin{array}{l}\text { Cross } \\
\text { sectional }\end{array}$ & $\begin{array}{l}\text { School } \\
\text { children }\end{array}$ & 157 & 162 & Amhara & KATO\&DWM & 319 & 243 & 3 \\
\hline $\begin{array}{l}\text { Alemayehu } \\
\text { 2015[90] }\end{array}$ & $\begin{array}{l}\text { Cross } \\
\text { sectional }\end{array}$ & $\begin{array}{l}\text { School } \\
\text { children }\end{array}$ & 201 & 183 & SNNPR & KATO\&DWM & 384 & 131 & 1 \\
\hline Ali 1999[91] & $\begin{array}{l}\text { Cross } \\
\text { sectional }\end{array}$ & $\begin{array}{l}\text { School } \\
\text { children }\end{array}$ & 161 & 121 & Oromia & KATO\&DWM & 282 & 170 & 0 \\
\hline Tulu 2016[65] & $\begin{array}{l}\text { Cross } \\
\text { sectional }\end{array}$ & $\begin{array}{l}\text { School } \\
\text { children }\end{array}$ & 251 & 241 & Oromia & DWM\&FEC & 492 & 44 & 0 \\
\hline $\begin{array}{l}\text { Unasho } \\
2013[71]\end{array}$ & $\begin{array}{l}\text { Cross } \\
\text { sectional }\end{array}$ & $\begin{array}{l}\text { School } \\
\text { children }\end{array}$ & 189 & 217 & SNNPR & DWM & 406 & 89 & 0 \\
\hline
\end{tabular}

DWM $=$ direct wet mount; FEC $=$ formal-ether: KATO = kato-katz: NNNPR $=$ Southern nations nationalities and peoples region, IPIs = intestinal parasitic infections: STHs = soil transmitted helminths: NA = not available: 


\begin{tabular}{|c|c|c|c|c|c|c|c|c|c|}
\hline $\begin{array}{l}\text { Name of first } \\
\text { author and } \\
\text { year }\end{array}$ & $\begin{array}{l}\text { Study } \\
\text { design }\end{array}$ & Population & Male & Female & Region & $\begin{array}{l}\text { Laboratory } \\
\text { method }\end{array}$ & $\begin{array}{l}\text { No. } \\
\text { sample }\end{array}$ & $\begin{array}{l}\text { Cases } \\
\text { for } \\
\text { IPls }\end{array}$ & $\begin{array}{l}\text { Quality } \\
\text { score }\end{array}$ \\
\hline $\begin{array}{l}\text { Belyhun } \\
2010[49]\end{array}$ & $\begin{array}{l}\text { Follow up } \\
\text { cohort }\end{array}$ & $\begin{array}{l}\text { Preschool } \\
\text { children }\end{array}$ & NA & NA & SNNPR & FEC & 905 & 292 & 3 \\
\hline Tulu 2014[38] & $\begin{array}{l}\text { Cross } \\
\text { sectional }\end{array}$ & $\begin{array}{l}\text { School } \\
\text { children }\end{array}$ & 172 & 168 & SNNPR & DWM\&FEC & 340 & 113 & 1 \\
\hline $\begin{array}{l}\text { Erosie } \\
2002[40]\end{array}$ & $\begin{array}{l}\text { Cross } \\
\text { sectional }\end{array}$ & $\begin{array}{l}\text { School } \\
\text { children }\end{array}$ & NA & NA & SNNPR & FEC & 421 & 69 & 1 \\
\hline $\begin{array}{l}\text { Tadesse } \\
2005[33]\end{array}$ & $\begin{array}{l}\text { Cross } \\
\text { sectional }\end{array}$ & $\begin{array}{l}\text { School } \\
\text { children }\end{array}$ & 271 & 144 & Oromia & FEC & 415 & 437 & 0 \\
\hline $\begin{array}{l}\text { Adamu } \\
2005[48]\end{array}$ & $\begin{array}{l}\text { Cross } \\
\text { sectional }\end{array}$ & $\begin{array}{l}\text { Preschool } \\
\text { children }\end{array}$ & 149 & 147 & Addis Ababa & DWM,FEC\&MZN & 296 & 571 & 0 \\
\hline $\begin{array}{l}\text { Jemaneh } \\
1999[92]\end{array}$ & $\begin{array}{l}\text { Cross } \\
\text { sectional }\end{array}$ & $\begin{array}{l}\text { School } \\
\text { children }\end{array}$ & 439 & 439 & Amhara & KATO & 878 & 165 & 0 \\
\hline $\begin{array}{l}\text { Dejenie } \\
2009[56]\end{array}$ & $\begin{array}{l}\text { Cross } \\
\text { sectional }\end{array}$ & $\begin{array}{l}\text { School } \\
\text { children }\end{array}$ & 1012 & 998 & Tigray & DWM & 2000 & 245 & 0 \\
\hline $\begin{array}{l}\text { Dejenie } 2010 \\
\text { [66] }\end{array}$ & $\begin{array}{l}\text { Cross } \\
\text { sectional }\end{array}$ & $\begin{array}{l}\text { School } \\
\text { children }\end{array}$ & 319 & 303 & Tigray & KATO & 622 & 263 & 0 \\
\hline $\begin{array}{l}\text { Nyantekyi } \\
2010[32]\end{array}$ & $\begin{array}{l}\text { Cross } \\
\text { sectional }\end{array}$ & $\begin{array}{l}\text { Preschool } \\
\text { children }\end{array}$ & 140 & 148 & SNNPR & KATO\&FEC & 288 & 282 & 1 \\
\hline $\begin{array}{l}\text { Legesse } \\
2010[52]\end{array}$ & $\begin{array}{l}\text { Cross } \\
\text { sectional }\end{array}$ & $\begin{array}{l}\text { School } \\
\text { children }\end{array}$ & 167 & 214 & Oromia & KATO\&FEC & 381 & 166 & 0 \\
\hline $\begin{array}{l}\text { Terefe } \\
2011[61]\end{array}$ & $\begin{array}{l}\text { Cross } \\
\text { sectional }\end{array}$ & $\begin{array}{l}\text { School } \\
\text { children }\end{array}$ & 218 & 201 & SNNPR & KATO & 419 & 285 & 1 \\
\hline $\begin{array}{l}\text { Debalke } \\
2013[93]\end{array}$ & $\begin{array}{l}\text { Cross } \\
\text { sectional }\end{array}$ & $\begin{array}{l}\text { School } \\
\text { children }\end{array}$ & 161 & 205 & Oromia & McMaster & 366 & 66 & 1 \\
\hline $\begin{array}{l}\text { Dejene } 2008 \\
\text { [94] }\end{array}$ & $\begin{array}{l}\text { Cross } \\
\text { sectional }\end{array}$ & $\begin{array}{l}\text { School } \\
\text { children }\end{array}$ & 481 & 319 & Tigray & FEC & 800 & 530 & 0 \\
\hline $\begin{array}{l}\text { Fekadu } 2008 \\
\text { [19] }\end{array}$ & $\begin{array}{l}\text { Cross } \\
\text { sectional }\end{array}$ & $\begin{array}{l}\text { School } \\
\text { children }\end{array}$ & 63 & 37 & Oromia & $\begin{array}{l}\text { Harada-Mori } \\
\text { (Test tube } \\
\text { culture) }\end{array}$ & 100 & 470 & 0 \\
\hline $\begin{array}{l}\text { Haileamlak } \\
\text { 2005[23] }\end{array}$ & $\begin{array}{l}\text { Cross } \\
\text { sectional }\end{array}$ & $\begin{array}{l}\text { Preschool } \\
\text { children }\end{array}$ & 487 & 437 & Oromia & DWM\&FEC & 924 & 74 & 1 \\
\hline $\begin{array}{l}\text { Jemaneh } \\
\text { 2001[42] }\end{array}$ & $\begin{array}{l}\text { Cross } \\
\text { sectional }\end{array}$ & $\begin{array}{l}\text { School } \\
\text { children }\end{array}$ & 282 & 405 & Amhara & KATO & 687 & 219 & 1 \\
\hline $\begin{array}{l}\text { Firdu } \\
2014[69]\end{array}$ & $\begin{array}{l}\text { Case- } \\
\text { control }\end{array}$ & Both & 135 & 95 & SNNPR & DWM,FEC\&MZN & 230 & 199 & 1 \\
\hline $\begin{array}{l}\text { Wale } \\
\text { 2014[62] }\end{array}$ & $\begin{array}{l}\text { Cross } \\
\text { sectional }\end{array}$ & $\begin{array}{l}\text { School } \\
\text { children }\end{array}$ & 206 & 196 & Amhara & DWM\&FEC & 402 & 562 & 1 \\
\hline $\begin{array}{l}\text { Teklemariam } \\
\text { 2014[67] }\end{array}$ & $\begin{array}{l}\text { Cross } \\
\text { sectional }\end{array}$ & $\begin{array}{l}\text { School } \\
\text { children }\end{array}$ & 252 & 228 & Tigray & FEC & 480 & 139 & 0 \\
\hline $\begin{array}{l}\text { Ayalew } \\
2011[27]\end{array}$ & $\begin{array}{l}\text { Cross } \\
\text { sectional }\end{array}$ & $\begin{array}{l}\text { School } \\
\text { children }\end{array}$ & 358 & 346 & Amhara & DWM\&FEC & 704 & 304 & 2 \\
\hline $\begin{array}{l}\text { Merid } \\
2001[30]\end{array}$ & $\begin{array}{l}\text { Cross } \\
\text { sectional }\end{array}$ & $\begin{array}{l}\text { School } \\
\text { children }\end{array}$ & NA & NA & SNNPR & DWM\&FEC & 150 & 465 & 0 \\
\hline $\begin{array}{l}\text { Assefa } \\
\text { 1998[112] }\end{array}$ & $\begin{array}{l}\text { Cross } \\
\text { sectional }\end{array}$ & $\begin{array}{l}\text { School } \\
\text { children }\end{array}$ & 479 & 219 & Amhara & FEC & 698 & 401 & 0 \\
\hline
\end{tabular}

DWM = direct wet mount; FEC $=$ formal-ether: KATO = kato-katz: NNNPR = Southern nations nationalities and peoples region, IPIs = intestinal parasitic infections: STHs = soil transmitted helminths: NA = not available: 


\begin{tabular}{|c|c|c|c|c|c|c|c|c|c|}
\hline $\begin{array}{l}\text { Name of first } \\
\text { author and } \\
\text { year }\end{array}$ & $\begin{array}{l}\text { Study } \\
\text { design }\end{array}$ & Population & Male & Female & Region & $\begin{array}{l}\text { Laboratory } \\
\text { method }\end{array}$ & $\begin{array}{l}\text { No. } \\
\text { sample }\end{array}$ & $\begin{array}{l}\text { Cases } \\
\text { for } \\
\text { IPls }\end{array}$ & $\begin{array}{l}\text { Quality } \\
\text { score }\end{array}$ \\
\hline $\begin{array}{l}\text { Roma } \\
\text { 1997[46] }\end{array}$ & $\begin{array}{l}\text { Cross } \\
\text { sectional }\end{array}$ & $\begin{array}{l}\text { School } \\
\text { children }\end{array}$ & 352 & 168 & SNNPR & FEC & 520 & 233 & 1 \\
\hline $\begin{array}{l}\text { Abera } \\
2013[95]\end{array}$ & $\begin{array}{l}\text { cross } \\
\text { sectional }\end{array}$ & $\begin{array}{l}\text { School } \\
\text { children }\end{array}$ & 397 & 381 & Amhara & KATO\&FEC & 772 & 311 & 3 \\
\hline $\begin{array}{l}\text { Kidane } \\
\text { 2014[113] }\end{array}$ & $\begin{array}{l}\text { Cross } \\
\text { sectional }\end{array}$ & $\begin{array}{l}\text { School } \\
\text { children }\end{array}$ & 177 & 207 & Tigray & DWM & 384 & 301 & 0 \\
\hline $\begin{array}{l}\text { Alamir } \\
2013[20]\end{array}$ & $\begin{array}{l}\text { Cross } \\
\text { sectional }\end{array}$ & $\begin{array}{l}\text { School } \\
\text { children }\end{array}$ & 192 & 207 & Amhara & DWM\&FEC & 399 & 104 & 0 \\
\hline $\begin{array}{l}\text { Kabeta } \\
\text { 2017[96] }\end{array}$ & $\begin{array}{l}\text { Cross } \\
\text { sectional }\end{array}$ & $\begin{array}{l}\text { Preschool } \\
\text { children }\end{array}$ & NA & NA & SNNPR & DWM\&FEC & 587 & 254 & 1 \\
\hline $\begin{array}{l}\text { Shumbej } \\
2015[97]\end{array}$ & $\begin{array}{l}\text { Cross } \\
\text { sectional }\end{array}$ & $\begin{array}{l}\text { Preschool } \\
\text { children }\end{array}$ & 165 & 212 & SNNPR & McMaster & 377 & 245 & 3 \\
\hline $\begin{array}{l}\text { Tadege } \\
\text { 2017[98] }\end{array}$ & $\begin{array}{l}\text { Cross } \\
\text { sectional }\end{array}$ & $\begin{array}{l}\text { School } \\
\text { children }\end{array}$ & 235 & 139 & SNNPR & FEC & 374 & 127 & 3 \\
\hline $\begin{array}{l}\text { Andualem } \\
\text { 2014[99] }\end{array}$ & $\begin{array}{l}\text { Cross } \\
\text { sectional }\end{array}$ & $\begin{array}{l}\text { School } \\
\text { children }\end{array}$ & 168 & 190 & Amhara & DWM\&FEC & 358 & 59 & 0 \\
\hline Reji 2011[45] & $\begin{array}{l}\text { Cross } \\
\text { sectional }\end{array}$ & $\begin{array}{l}\text { School } \\
\text { children }\end{array}$ & NA & NA & , Oromia & KATO & 358 & 52 & 1 \\
\hline $\begin{array}{l}\text { Alemu } \\
2014[64]\end{array}$ & $\begin{array}{l}\text { Cross } \\
\text { sectional }\end{array}$ & $\begin{array}{l}\text { School } \\
\text { children }\end{array}$ & 211 & 194 & SNNPR & KATO & 405 & 110 & 0 \\
\hline $\begin{array}{l}\text { Samuel } \\
2015[114]\end{array}$ & $\begin{array}{l}\text { Cross } \\
\text { sectional }\end{array}$ & $\begin{array}{l}\text { School } \\
\text { children }\end{array}$ & NA & NA & Oromia & FEC & 375 & 42 & 3 \\
\hline $\begin{array}{l}\text { Teshale } \\
\text { 2018[100] }\end{array}$ & $\begin{array}{l}\text { Cross } \\
\text { section }\end{array}$ & $\begin{array}{l}\text { School } \\
\text { children }\end{array}$ & 240 & 170 & Tigray & KATO & 410 & 58 & 1 \\
\hline $\begin{array}{l}\text { Tekeste } \\
2013[60]\end{array}$ & $\begin{array}{l}\text { Cross } \\
\text { sectional }\end{array}$ & $\begin{array}{l}\text { School } \\
\text { children }\end{array}$ & 170 & 156 & Amhara & KATO & 326 & 109 & 2 \\
\hline $\begin{array}{l}\text { Sitotaw } 2019 \\
\text { [60] }\end{array}$ & $\begin{array}{l}\text { Cross } \\
\text { sectional }\end{array}$ & $\begin{array}{l}\text { School } \\
\text { children }\end{array}$ & 216 & 190 & Amhara & DWM\&FEC & 406 & 235 & 3 \\
\hline $\begin{array}{l}\text { Elfu } 2018 \\
\text { [101] }\end{array}$ & $\begin{array}{l}\text { Cross } \\
\text { sectional }\end{array}$ & $\begin{array}{l}\text { School } \\
\text { children }\end{array}$ & 1129 & 1261 & Amhara & DWM\&FEC & 2390 & 684 & 3 \\
\hline $\begin{array}{l}\text { Molla } 2018 \\
\text { [104] }\end{array}$ & $\begin{array}{l}\text { Cross } \\
\text { sectional }\end{array}$ & $\begin{array}{l}\text { School } \\
\text { children }\end{array}$ & 245 & 198 & SNNPR & KATO & 443 & 239 & 3 \\
\hline $\begin{array}{l}\text { Weldesenbet } \\
2019 \text { [107] }\end{array}$ & $\begin{array}{l}\text { Cross } \\
\text { sectional }\end{array}$ & $\begin{array}{l}\text { School } \\
\text { children }\end{array}$ & 349 & 251 & SNNPR & KATO & 600 & 57 & 3 \\
\hline $\begin{array}{l}\text { Eyamo } 2019 \\
\text { [102] }\end{array}$ & $\begin{array}{l}\text { Cross } \\
\text { sectional }\end{array}$ & $\begin{array}{l}\text { School } \\
\text { children }\end{array}$ & 199 & 185 & SNNPR & DWM & 384 & 260 & 3 \\
\hline $\begin{array}{l}\text { Tadesse } \\
2020[106]\end{array}$ & $\begin{array}{l}\text { Cross } \\
\text { sectional }\end{array}$ & $\begin{array}{l}\text { School } \\
\text { children }\end{array}$ & 204 & 213 & Oromia & DWM\&FEC & 422 & 131 & 2 \\
\hline $\begin{array}{l}\text { Gadisa } 2019 \\
\text { [16] }\end{array}$ & $\begin{array}{l}\text { Cross } \\
\text { sectional }\end{array}$ & $\begin{array}{l}\text { Preschool } \\
\text { children }\end{array}$ & 242 & 319 & Oromia & DWM\&FEC & 561 & 216 & 3 \\
\hline Zenu [110] & $\begin{array}{l}\text { Cross } \\
\text { sectional }\end{array}$ & $\begin{array}{l}\text { School } \\
\text { children }\end{array}$ & 284 & 28 & Oromia & DWM\&FEC & 312 & 208 & 3 \\
\hline Shumbej[109] & $\begin{array}{l}\text { Cross } \\
\text { sectional }\end{array}$ & $\begin{array}{l}\text { School } \\
\text { children }\end{array}$ & 350 & 247 & SNNPR & KATO & 597 & 141 & 3 \\
\hline
\end{tabular}

DWM $=$ direct wet mount; FEC $=$ formal-ether: KATO = kato-katz: NNNPR $=$ Southern nations nationalities and peoples region, IPIs = intestinal parasitic infections: STHs = soil transmitted helminths: NA = not available: 


\begin{tabular}{|c|c|c|c|c|c|c|c|c|c|}
\hline $\begin{array}{l}\text { Name of first } \\
\text { author and } \\
\text { year }\end{array}$ & $\begin{array}{l}\text { Study } \\
\text { design }\end{array}$ & Population & Male & Female & Region & $\begin{array}{l}\text { Laboratory } \\
\text { method }\end{array}$ & $\begin{array}{l}\text { No. } \\
\text { sample }\end{array}$ & $\begin{array}{l}\text { Cases } \\
\text { for } \\
\text { IPls }\end{array}$ & $\begin{array}{l}\text { Quality } \\
\text { score }\end{array}$ \\
\hline Workneh[108] & $\begin{array}{l}\text { Cross } \\
\text { sectional }\end{array}$ & $\begin{array}{l}\text { School } \\
\text { children }\end{array}$ & 194 & 146 & Amhara & KATO & 340 & 51 & 3 \\
\hline
\end{tabular}

DWM $=$ direct wet mount; FEC $=$ formal-ether: KATO = kato-katz: NNNPR = Southern nations nationalities and peoples region, IPIs = intestinal parasitic infections: $\mathrm{STH}=$ soil transmitted helminths: $\mathrm{NA}=$ not available:

\section{Pooled Prevalence Estimate Of Intestinal Parasites And Heterogeneity}

Eighty studies (88) studies consisting of 61,690 preschool and school-age children reported the proportion of intestinal parasitic infections. Out of these, 29,311 children were infected with one or more species of intestinal parasites giving the pooled prevalence estimate of $48 \%(95 \% \mathrm{Cl}: 43-53 \%)$ with considerable heterogeneity $\left(\chi 2=17303.64, \mathrm{P}<0.001 ; I^{2}=99.50 \%\right)$. The prevalence of intestinal parasitic infection was 53\%(95\% Cl: $38-67 \%), 50 \%(95 \% \mathrm{Cl}: 44-57 \%), 45 \% \%(95 \% \mathrm{Cl}: 35-54 \%)$ and $43 \% \%(95 \% \mathrm{Cl}: 29-58 \%)$ in Southern Nations Nationalities and Peoples Region (SNNPR), Amhara, Oromia, and Tigray regions, respectively (Fig. 2). We also did subgroupanalysis to see the influence of study design on prevalence. Interestingly enough, the prevalence was $48 \%$ ( $95 \%$ Cl: $43-53 \%$ ) for cross ectional study sdesign and therefore, the inclusion of other study designs has no influence on the overall rate of infection ( not shown).

\section{Overall Prevalence Estimate Of Soil-transmitted Helminthes (sths) And Heterogeneity}

Soil-transmitted helminths detected in the studies were Ascaris lumbricoides, Hookworms, Trichuris trichiura and Strongyloides stercoralis. A total of 19,678 of the 61,690 children examined during the period under review were infected with one or more species of STHs yielding an overall prevalence of $33 \%(95 \% \mathrm{Cl}: 28-38 \%)$ with substantial heterogeneity $\left(X 2=30360.02, P<0.001 ; I^{2}=99.71 \%\right)$ (Fig. 3). The asymmetry of funnel plot visual inspection (Fig. 4) showed that the presence of publication bias which was statistically confirmed by Egger's test $(\beta=16.7$, [95\% Cl: 10.7-22.5], $p<0.001$ and Begg's test $p<0.001$.

We did meta-regression analyses to search for the sources of heterogeneity. A univariate meta-regression between prevalence of STHs and age of children showed statistically significant correlation $(P=0.003, F i g .5)$. However, year of publications, $(P=0.076)$, regional states $(p=0.70)$ and study design $(p=0.23)$ did not show a statistically significant correleation as shown in Table 2.

Table 2

Univariate meta-regression of factors related to the heterogeneity of soil-transmitted helminths among Ethiopian children, 2020

\begin{tabular}{|llll|}
\hline Variables & $\boldsymbol{\beta}$-Coefficient & $\mathbf{9 5 \%} \mathbf{C l}$ & $\boldsymbol{p}$-values \\
\hline Regional states & -0.03 & -0.18 to 0.12 & 0.70 \\
\hline Year of publication & -0.04 & -0.004 to 0.08 & 0.076 \\
\hline Age & 0.73 & 0.25 to 1.2 & 0.003 \\
\hline Study design & -0.45 & -1.2 to 0.30 & 0.23 \\
\hline Cl = confidence interval & & \\
\hline
\end{tabular}

Sub-group analysis based on geographical region and age of children

Subgroup analysis showed that the prevalence of STHs was $44 \%(95 \% \mathrm{Cl}: 31-58 \%)$ in SNNPR, $34 \%(95 \% \mathrm{Cl}: 28-41 \%)$ in Amhara region, $31 \%(95 \% \mathrm{Cl}: 19 \%-43 \%)$ in Oromia region and $10 \%(95 \% \mathrm{Cl}: 7 \%-12 \%)$ in Tigray region as shown in Fig. 6 . The age related prevalence was $51 \%$ (95\% Cl: $45-56 \%)$ in school-age children and $32 \%(95 \% \mathrm{Cl}: 20-44 \%)$ in preschool-age children $(p=0.003)$ as shown in Fig. 7. Subgroup analysis by publication year showed that the pooled prevalence of STHs between 1995-2012 years was $44 \%$ (95\% Cl:30-57\%) while, it was 30\% (95\%Cl: 25-34\%) for studies conducted between 2013-2020 years (Fig. 8). In summary, STHs were more common in SNNPR among school-age children in studies published between 1990-2012 as shown in Table 3. We 
performed subgroup analysis based on study design and the result showed that the prevalence of STHs was 34\% (95\% Cl:29-39\%) for cross sectional study, $25 \%$ (95\% Cl:23-28\%), 4\% (95\% Cl:3-5\%) for prospective study and 20\% (95\% Cl:15-26\%) for case-control study (not shown). This indicates that the overall prevalence is almost the same as the prevalence of studies with cross sectional study design and was not affected by other study designs.

Table 3

Prevalence of soil-transmitted helminths (STHs) by region, age of children and year of publication among Ethiopian Children, 2020

\begin{tabular}{|c|c|c|c|c|c|c|c|}
\hline \multirow[t]{2}{*}{ Variables } & \multirow[t]{2}{*}{ No. of studies } & \multirow[t]{2}{*}{ Sample } & \multirow[t]{2}{*}{ cases } & \multirow{2}{*}{$\begin{array}{l}\text { Prevalence } \\
(95 \% \mathrm{Cl})\end{array}$} & \multicolumn{2}{|c|}{ Heterogeneity } & \multirow[t]{2}{*}{ P-value } \\
\hline & & & & & $\mathbf{Q}$ & $\mathrm{I}^{2}(\%)$ & \\
\hline \multicolumn{8}{|l|}{ Region } \\
\hline Addis Ababa city & 1 & 296 & 10 & $3(2-6 \%)$ & - & - & - \\
\hline Amhara region & 36 & 36809 & 12374 & $34(28-41 \%)$ & 8325.55 & 99.58 & $P<0.001$ \\
\hline Benishangul-Gumuz region & 1 & 422 & 35 & $8(6-11 \%)$ & - & - & - \\
\hline Oromia region & 20 & 9119 & 2780 & $31(19-43 \%)$ & 9070.41 & 99.79 & $P<0.001$ \\
\hline South Region & 22 & 9379 & 3869 & $44(31-58 \%)$ & 7621.83 & 99.72 & $P<0.001$ \\
\hline Tigray region & 8 & 5665 & 610 & $10(7-12 \%$ & 66.61 & 89.49 & $P<0.001$ \\
\hline \multicolumn{8}{|l|}{ Age } \\
\hline School & 73 & 55731 & 18225 & $36(31-42 \%)$ & 27820.22 & 99.74 & $P<0.001$ \\
\hline Preschool & 13 & 5577 & 1408 & $20(11-29 \%)$ & 1764.59 & 99.32 & $P<0.001$ \\
\hline Both & 2 & 382 & 45 & $6(4-9 \%)$ & - & - & - \\
\hline \multicolumn{8}{|l|}{ Year of publication } \\
\hline 1997-2012 & 22 & 12831 & 4607 & $44(30-57 \%)$ & 14221.00 & 99.85 & $P<0.001$ \\
\hline $2013-2020$ & 66 & 48859 & 15071 & $30(25-34 \%)$ & 15324.02 & 99.58 & $P<0.001$ \\
\hline Overall & 88 & 61690 & 19678 & $33(28-38 \%)$ & 30360.02 & 99.71 & $P<0.001$ \\
\hline
\end{tabular}

\section{Prevalence Of Sths By Species}

\section{Ascaris lumbricoides}

Eighty five studies consisting of 58, 234 children have reported that the pooled prevalence of $A$. lumbricoides was 17\% (95\% Cl: 15 to $19 \%)$ with substantial heterogeneity $\left(X 2=8961.94, \mathrm{P}<0.001 ; \mathrm{I}^{2}=99.06 \%\right)$. The prevalence was $27 \%(95 \% \mathrm{Cl}: 21$ to $34 \%)$ in SNNPR, 14\%(95\% Cl: 11 to $17 \%$ ) in Amhara region, 15\%(95\% Cl: 11 to 19\%) in Oromia region and 6\%( $95 \% \mathrm{Cl}: 3$ to $8 \%$ ) in Tigray region [supplementary file 1]. The age related prevalence of $A$. lumbricoides was $18 \%(95 \% \mathrm{Cl}: 15$ to $20 \%)$ in school-age children and $12 \%$ $(95 \% \mathrm{Cl}: 8$ to $17 \%)$ in preschool-age children ( $\mathrm{p}=0.06)$. The pooled prevalence of $A$. lumbricoides was $25 \%(95 \% \mathrm{Cl}: 19$ to $31 \%)$ in studies published between 1997-2012 years and 14\% (95\% Cl: 12 to 16\%) beween 2013-2020 years. A univariate meta-regression between prevalence and year of publications showed statistically significant correlation $(\beta=-0.49$ ( $95 \% \mathrm{Cl}:-1.1$ to $-0.07, \mathrm{P}=0.035$, Supplementary file 2$)$. However, regional states ( $\beta$ : $0.046,95 \% \mathrm{Cl}:-0.12$ to $0.0 .22, \mathrm{p}=0.58)$ and age of children $(\beta: 0.52,95 \% \mathrm{Cl}:-0.02$ to $1.1, p=0.06$ ) did not show a statistically significant relationship. Therefore, Ascaris lumbricoides was the most predominant species of STHs among Ethiopian children and significant decline in prevalence was observed over two decades (from late 1990s to 2020) (Table 4). 
Table 4

Pooled prevalence of species specific Ascaris lumbricoides by region, age and year of publication among Ethiopian children, 2020

\begin{tabular}{|c|c|c|c|c|c|c|c|}
\hline \multirow[t]{2}{*}{ Variables } & \multirow[t]{2}{*}{ No. of studies } & \multirow[t]{2}{*}{ Sample } & \multirow[t]{2}{*}{ cases } & \multirow{2}{*}{$\begin{array}{l}\text { Prevalence } \\
(95 \% \mathrm{Cl})\end{array}$} & \multicolumn{2}{|c|}{ Heterogeneity } & \multirow[t]{2}{*}{ P-value } \\
\hline & & & & & $\mathbf{Q}$ & $\mathrm{I}^{2}(\%)$ & \\
\hline \multicolumn{8}{|l|}{ Region } \\
\hline Addis Ababa city & 1 & 296 & 8 & $3(1-5 \%)$ & - & - & - \\
\hline Amhara region & 35 & 34419 & 5311 & $14(11-17 \%)$ & 3356.20 & 98.99 & $P<0.001$ \\
\hline Oromia region & 19 & 8475 & 1271 & $15(11-19 \%)$ & 934.49 & 98.07 & $P<0.001$ \\
\hline South Region & 22 & 9379 & 2374 & $27(21-34 \%)$ & 4265.90 & 99.51 & $P<0.001$ \\
\hline Tigray region & 8 & 5665 & 375 & $6(3-8 \%)$ & 148.81 & 95.30 & $P<0.001$ \\
\hline \multicolumn{8}{|l|}{ Age } \\
\hline School & 70 & 52275 & 8509 & $18(15-20 \%)$ & 7820.37 & 99.12 & $P<0.001$ \\
\hline Preschool & 13 & 5577 & 822 & $12(8-17 \%)$ & 892.82 & 98.66 & $P<0.001$ \\
\hline Both & 2 & 382 & 8 & $2(1-4 \%)$ & - & - & - \\
\hline \multicolumn{8}{|l|}{ Year of publication } \\
\hline $1990-2012$ & 22 & 12831 & 2841 & $25(19-31 \%)$ & 4111.93 & 99.49 & $P<0.001$ \\
\hline $2013-2020$ & 63 & 45403 & 6498 & $14(12-16 \%)$ & 4838.10 & 98.72 & $P<0.001$ \\
\hline Overall & 85 & 58234 & 9339 & $17(15-19 \%)$ & 8961.94 & 99.06 & $P<0.001$ \\
\hline
\end{tabular}


Table 5

Pooled prevalence of species specific Trichuris trichiura by region, age and year of publication among Ethiopian children, 2020

\begin{tabular}{|c|c|c|c|c|c|c|c|}
\hline \multirow[t]{2}{*}{ Variables } & \multirow[t]{2}{*}{ No. of studies } & \multirow[t]{2}{*}{ Sample } & \multirow[t]{2}{*}{ cases } & \multirow{2}{*}{$\begin{array}{l}\text { Prevalence } \\
(95 \% \mathrm{Cl})\end{array}$} & \multicolumn{2}{|c|}{ Heterogeneity } & \multirow[t]{2}{*}{ P-value } \\
\hline & & & & & $\mathbf{Q}$ & $\mathrm{I}^{2}(\%)$ & \\
\hline \multicolumn{8}{|l|}{ Region } \\
\hline Addis Ababa city & 1 & 296 & 2 & $1(0-2 \%)$ & - & - & - \\
\hline Amhara region & 28 & 31555 & 1186 & $4(3-4 \%)$ & 528.28 & 94.89 & $P<0.001$ \\
\hline Oromia region & 19 & 8738 & 1089 & $10(8-13 \%)$ & 1282.36 & 98.60 & $P<0.001$ \\
\hline South Region & 22 & 9379 & 949 & $11(9-13 \%)$ & 1728.08 & 98.78 & $P<0.001$ \\
\hline Tigray region & 6 & 4886 & 86 & $1(0-2 \%)$ & 61.86 & 91.92 & $P<0.001$ \\
\hline \multicolumn{8}{|l|}{ Age } \\
\hline School & 63 & 49327 & 3093 & $7(6-8 \%)$ & 3318.18 & 98.13 & $P<0.001$ \\
\hline Preschool & 12 & 5267 & 211 & $4(2-6 \%)$ & 443.50 & 97.52 & $P<0.001$ \\
\hline Both & 1 & 260 & 8 & $3(2-6 \%)$ & - & - & - \\
\hline \multicolumn{8}{|l|}{ Year of publication } \\
\hline 1990-2012 & 20 & 11786 & 1374 & $14(12-17 \%)$ & 2176.26 & 99.13 & $P<0.001$ \\
\hline $2013-2020$ & 56 & 43068 & 1938 & $4(4-5 \%)$ & 1569.21 & 96.50 & $P<0.001$ \\
\hline Overall & 76 & 58234 & 9339 & $6(6-7 \%)$ & 3766.86 & 98.01 & $P<0.00$ \\
\hline
\end{tabular}

\section{Trichuris trichiura}

Seventy six studies included of 54,854 children have reported that the pooled prevalence of Trichuris trichiura was $6 \%$ ( $95 \% \mathrm{Cl}$ : 6 to $7 \%)$ with considerable heterogeneity $\left(X 2=3766.86, \mathrm{P}<0.001 ; I^{2}=98.01 \%\right)$. The pooled prevalence was $11 \%(95 \% \mathrm{Cl}: 11-13 \%)$ in SNNPR, 10\% (95\% Cl: 8-13\%) in Oromia region, 4\% (95\% Cl: 3-4\%) in Amhara region and 1\% (95\% Cl: 0-2\%) in Tigray region and 1 [supplementary file 3]. The age related prevalence was also $7 \%(95 \% \mathrm{Cl}: 6 \%$ to $8 \%$,) among school-age children and $4 \%(95 \% \mathrm{Cl}$ : $2-6 \%)$ among preschool-age children $(\mathrm{p}=0.24)$. The pooled prevalence of $T$. trichura was $14 \%(95 \% \mathrm{Cl}: 12-17 \%)$ in studies conducted between 1997-2013 years and 4\% (95\% Cl: 4-24\%) between 2013-2020 years. A univariate meta-regression between prevalence and year of publications showed statistically significant correlation ( $B=-0.78,95 \% \mathrm{Cl}:-1.5$ to $-0.069, p=0.03$, Supplementary file 4). However, regional states ( $\beta$ : $0.003,95 \% \mathrm{Cl}:-0.22$ to $0.0 .23, p=0.97)$ and age of children $(\beta: 0.46,95 \% \mathrm{Cl}:-0.29$ to $1.2, p=0.23)$ did not show a statistically significant relationship. The bottom line is that the rate of infection of Trichuris trichiura among Ethiopian children decreased significantly after starting of MDA as detailed in Fig. 5 .

\section{Hookworms}

Seventy six studies consisting of 54,854 children have also reported the pooled prevalence of Hookworms. Hence, the pooled prevalence on analysis was $12 \%(95 \% \mathrm{Cl}: 10$ to $13 \%)$ with substantial heterogeneity $\left(X 2=7920.16, P<0.001 ; I^{2}=99.05 \%\right)$. The pooled prevalence of hookworms was $12 \%(95 \% \mathrm{Cl}$ : $9-15 \%)$ in SNNPR, $16 \%(95 \% \mathrm{Cl}$ : $13-19 \%)$ in Amhara region, $6 \%(95 \% \mathrm{Cl}$ : 5-8\%) in Oromia region, and $3 \%$ (95\% Cl: 2-4\%) in Tigray region as shown in supplementary file 5 . The age related prevalence of hookworms was $13 \%$ (95\% Cl: $11 \%$ to $15 \%)$ among school-age children and $2 \%(95 \% \mathrm{Cl}: 1-3 \%)$ among preschool-age children $(p=0.01)$. The pooled prevalence of hookworms was $13 \%(95 \% \mathrm{Cl}$ : $9-15 \%)$ in studied conducted between $1997-2012$ years and $11 \%(95 \% \mathrm{Cl}$ : 9-13\%) in studies between 2013-2020 years.

A univariate meta-regression between prevalence and age of children showed statistically significant correlation $(B=1.03,95 \% \mathrm{Cl}$ : 0.27 to $1.8, p=0.01$, supplementary file $6 \mathrm{~A})$. In Addition,meta-regression of the prevalence and regional states $(\beta:-0.20,95 \% \mathrm{Cl}:-0.40$ 
to $-0.0 .005, p=0.045$, supplementary file $6 \mathrm{~B}$ ) revealed a significant correlation. However, year of publication ( $\beta$ : $-0.09,95 \% \mathrm{Cl}:-0.79$ to $0.61, p=0.81$ ) did not show a statistically significant relationship. In summary, Hookworms were more prevalent among children in Amhara region compared to other regions and among school-age children compared to preschool-age children ( Table 6).

Table 6

Pooled prevalence of species specific Hookworms by region, age and year of publication among Ethiopian children, 2020

\begin{tabular}{|c|c|c|c|c|c|c|c|}
\hline \multirow[t]{2}{*}{ Variables } & \multirow[t]{2}{*}{ No. of studies } & \multirow[t]{2}{*}{ Sample } & \multirow[t]{2}{*}{ cases } & \multirow{2}{*}{$\begin{array}{l}\text { Prevalence } \\
(95 \% \mathrm{Cl})\end{array}$} & \multicolumn{2}{|c|}{ Heterogeneity } & \multirow[t]{2}{*}{ P-value } \\
\hline & & & & & $\mathbf{Q}$ & $\mathrm{I}^{2}(\%)$ & \\
\hline \multicolumn{8}{|l|}{ Region } \\
\hline Benishangul-Gumuz & 1 & 422 & 35 & $8(6-11 \%)$ & - & - & - \\
\hline Amhara region & 32 & 35678 & 6171 & $16(13-19 \%)$ & 5256.02 & 99.41 & $P<0.001$ \\
\hline Oromia region & 15 & 6763 & 434 & $6(5-8 \%)$ & 392.85 & 96.44 & $P<0.001$ \\
\hline South Region & 20 & 8761 & 950 & $12(9-15 \%)$ & 793.61 & 97.61 & $P<0.001$ \\
\hline Tigray region & 8 & 5665 & 144 & $3(2-4 \%)$ & 96.14 & 92.72 & $P<0.001$ \\
\hline \multicolumn{8}{|l|}{ Age } \\
\hline School & 67 & 53289 & 7648 & $13(11-15 \%)$ & 7814.96 & 99.16 & $P<0.001$ \\
\hline Preschool & 8 & 3740 & 102 & $2(1-3 \%)$ & 33.50 & 79.10 & $P<0.001$ \\
\hline Both & 1 & 260 & 4 & $2(1-4 \%)$ & - & - & - \\
\hline \multicolumn{8}{|l|}{ Year of publication } \\
\hline $1990-2012$ & 19 & 11253 & 1534 & $13(9-15 \%)$ & 1346.55 & 98.66 & $P<0.001$ \\
\hline $2013-2020$ & 57 & 46036 & 6220 & $11(9-13 \%)$ & 6088.71 & 99.08 & $P<0.001$ \\
\hline Overall & 76 & 57289 & 7754 & $12(10-13 \%)$ & 7920.16 & 99.05 & $P<0.001$ \\
\hline
\end{tabular}

\section{Strongyloides stercoralis}

Twenty six studies consisting of 11,748 children have reported that the pooled prevalence of Strongyloides stercoralis was $1 \%$ (95\% Cl: 1 to $2 \%)$. The pooled prevalence of Strongyloides stercoralis was 3\% (95\%Cl: 1-4\%) in Amhara region, $1 \%(95 \% \mathrm{Cl}: 1-2 \%)$ in SNNPR, $1 \%(95 \% \mathrm{Cl}: 0-1 \%)$ in Oromia region and $0 \%(95 \% \mathrm{Cl}: 0-1 \%)$ in Tigray region as shown in supplementary file 7. The prevalence was $1 \%(95 \% \mathrm{Cl}: 1 \%$ to $2 \%)$ in school-age children. The pooled prevalence of Strongyloides stercoralis was $1 \%(95 \% \mathrm{Cl}: 1-2 \%)$ in studies done between 1997-2012 years and 2\% (95\% Cl: 1-2\%) between 2013-2020.

A univariate meta-regression between prevalence and regional states showed statistically significant correlation $(B=-0.30,95 \% \mathrm{Cl}$ : -0.56 to $-0.03, p=0.03$, supplementary file 8 ). However, year of publication ( $\beta:-0.17,95 \% \mathrm{Cl}:-0.70$ to $1.0, p=0.70)$ and age of children ( $\beta$ : $-0.02,95 \% \mathrm{Cl}:-0.96$ to $0.92, p=0.97)$ did not show a statistically significant relationship. Therefore, Strongyloides stercoralis is more common among children in Amhara region compared to other regions (Table 7). For further details, the summary of species specific STHs presented on Table 8. 
Table 7

Pooled prevalence of species specific Strongyloides stercoralis by region, age and year of publication among Ethiopian children, 2020

\begin{tabular}{|c|c|c|c|c|c|c|c|}
\hline \multirow[t]{2}{*}{ Variables } & \multirow[t]{2}{*}{ No. of studies } & \multirow[t]{2}{*}{ Sample } & \multirow[t]{2}{*}{ cases } & \multirow{2}{*}{$\begin{array}{l}\text { Prevalence } \\
(95 \% \mathrm{Cl})\end{array}$} & \multicolumn{2}{|c|}{ Heterogeneity } & \multirow[t]{2}{*}{ P-value } \\
\hline & & & & & $\mathbf{Q}$ & $\mathrm{I}^{2}(\%)$ & \\
\hline \multicolumn{8}{|l|}{ Region } \\
\hline Amhara region & 11 & 5131 & 163 & $3(1-4 \%)$ & 116.39 & 91.41 & $P<0.001$ \\
\hline Oromia region & 5 & 1566 & 10 & $1(0-1 \%)$ & 2.13 & 00.00 & $P=0.71$ \\
\hline South Region & 7 & 3149 & 54 & $1(1-2 \%)$ & 35.77 & 83.23 & $P<0.001$ \\
\hline Tigray region & 3 & 1902 & 9 & $0(0-1 \%)$ & - & - & - \\
\hline \multicolumn{8}{|l|}{ Age } \\
\hline School & 23 & 10134 & 204 & $1(1-2 \%)$ & 150.65 & 85.40 & $P<0.001$ \\
\hline Preschool & 2 & 1492 & 30 & $0(0-1 \%)$ & - & - & - \\
\hline Both & 1 & 122 & 2 & $2(0-6 \%)$ & - & - & - \\
\hline \multicolumn{8}{|c|}{ Year of publication } \\
\hline $1990-2012$ & 11 & 5653 & 79 & $1(1-2 \%)$ & 141.55 & 90.11 & $P<0.001$ \\
\hline $2013-2020$ & 15 & 6095 & 157 & $2(1-2 \%)$ & 6088.71 & 72.96 & $P<0.001$ \\
\hline Overall & 26 & 11748 & 236 & $1(1-2 \%)$ & 179.49 & 86.07 & $P<0.001$ \\
\hline
\end{tabular}

Table 8

summary of species-specific pooled prevalence estimates of STHs among Ethiopian children, 2020

\begin{tabular}{|c|c|c|c|c|c|c|c|c|}
\hline \multirow[t]{2}{*}{ Parasites } & \multirow{2}{*}{$\begin{array}{l}\text { Number of } \\
\text { studies }\end{array}$} & \multirow{2}{*}{$\begin{array}{l}\text { Sample } \\
\text { size }\end{array}$} & \multirow[t]{2}{*}{ positives } & \multirow{2}{*}{$\begin{array}{l}\text { Prevalence } \\
(\%)\end{array}$} & \multirow[t]{2}{*}{$95 \% \mathrm{Cl}$} & \multicolumn{2}{|c|}{ Heterogeneity } & \multirow{2}{*}{$\begin{array}{l}\mathrm{p} \text { - } \\
\text { value }\end{array}$} \\
\hline & & & & & & $\mathbf{Q}$ & $\mathrm{P}^{2}(\%)$ & \\
\hline Ascaris lumbricoides & 85 & 58234 & 9339 & 17 & $\begin{array}{l}15- \\
19 \%\end{array}$ & 8961.94 & 99.06 & $\begin{array}{l}P< \\
0.001\end{array}$ \\
\hline Trichuris trichiura & 76 & 54854 & 3312 & 6 & $6-7 \%$ & 3766.86 & 98.01 & $\begin{array}{l}P< \\
0.001\end{array}$ \\
\hline Hookworms & 76 & 57289 & 7754 & 12 & $\begin{array}{l}10- \\
13 \%\end{array}$ & 7920.16 & 99.05 & $\begin{array}{l}P< \\
0.001\end{array}$ \\
\hline $\begin{array}{l}\text { Strongyloides } \\
\text { stercoralis }\end{array}$ & 26 & 11748 & 236 & 1 & $1-2 \%$ & 179.49 & 86.07 & $\begin{array}{l}\mathrm{P}< \\
0.001\end{array}$ \\
\hline
\end{tabular}

\section{Intesnity Of Sths Infection}

Only 13 out of 88 studies included 5, 676 children reported about intensity of infection of STHs. Low intensity of infection of A. lumbricoides was observed in $16 \%$ (95\% Cl: 10 to $21 \%$, supplementary file 9 ) children. Moderate and high intensity of infections of $A$. lumbricoides were observed in 13\% (95\% Cl: 7 to 19\%, supplementary file 10) and 6\% (95\% Cl: 2 to $11 \%$, supplementary file 11 ) of children, respectively. Low, moderate and high intensity of infections of T. trichura were observed in 16\% (95\% Cl: 12 to $20 \%$, supplementary 12), 3\%(95\% Cl: 2 to $4 \%$, supplementary file 13), $1 \%$ (95\% Cl: 1 to $2 \%$, supplementary file 14) children, respectively. This reviewe also showed that low, moderate and high intensity of infections of Hookworms were recorded in 20\% (95\% Cl: 10 to $29 \%$, supplementary file 15$), 4 \%$ ( $95 \% \mathrm{Cl}: 2$ to $6 \%$, supplementary file 16$)$ and $5 \%$ ( $95 \% \mathrm{Cl}: 0$ to $11 \%$, supplementary file 17$)$ children, respectively. 


\section{Regional distribution of eligible studies and risk zones (RZs) for STHs infections}

The highest numbers of studies were reported from Amhara 36 (40.90\%) and SNNPR 22(25\%). These were followed by the Oromia region 20 (22.7\%), Tigray 8 (9.1\%), Benishangul-Gumuz region and Addis Ababa city each with one (1.1\%) study. None of the regions is classified as High Risk Zone (HRZ) according to world health organization (WHO) risk classification. SNNPR, Amhara and Oromia regions recorded STH prevalence of $44 \%, 34 \%, 31 \%$, respectively and are classified as moderate risk zones (MRZs) while, the rest of the regions and cities recorded prevalence estimates ranging between $1-10 \%$ and are classified as Low Risk Zones (LRZs).

\section{Discussions}

The purpose of current systematic review and meta- analysis of STHs infections data analysis among Ethiopian children was to measure the impact of the ongoing control and preventive measures in the country and support the efforts undertaken to control and eliminate neglected tropical diseases (NTDs) by nurturing or supplementing useful national epidemiological data. Such studies have the potential to guide concerned bodies to focus their efforts in highly endemic areas. Although several studies have been published from different regions of Ethiopia on STHs with the earliest scientific literature dating back 1990s, the data on STHs infections remains unorganized and scattered. Therefore, organizing and locating information has the potential to inform and develop a comprehensive approach to control STH infections and target highly endemic areas with greater urgency.

The overall pooled estimate of STHs (33\%) observed in the present review is in line with the study from south America 27.1\% [111], but higher than study done in Iran (9.48\%) [112] and Côte d'Ivoire (19.1\%) [113]. The prevalence is lower than study from Nigeria (54.8\%) [114] and repors from other Sub-Sharan African countres (52.4-65.8\%). The variation between the findings might be attributed to differences in sensitivity and specificity of diagnostic methodology, environmental factors such as soil moisture, humidity, temperature and level of participants' hygiene and sanitation. In addition, our review included more recent surveys that the ongoing MDA and Sustainable water, sanitation, and hygiene (WASH) programs decreased the prevalence of STHs in Ethiopian children unlike the systematic review from Nigeria which included old studies from the year 1985 [114]

Subgroup analysis of the current review also showed that STHs are more common in SNNPR, Amhara and Oromia regions, although variation among the regional states was not statistically siginificant $(p=0.70)$. The majority of these infections are related to low standard of living, poor socioeconomic status, poor personal hygiene, and poor environmental sanitation. The higher prevalence of STHs infection among children in SNNPR, Amhara and Oromia regions might be also related to the high rainfall, forest and low temperature which favors the survival and transmission of the helminths in these regions. The lowest prevalence in Addis Ababa, capital city of Ethiopia, might to be due to advanced life style, good personal hygiene and good quality of life.

Our review suggests that the risk of STH infections has decreased from 44-30\% in studies conducted between 1997-2012 and 2013-2020 respectively, although the decline is not statistically significant $(p=0.45)$. On one hand, prevalence might have declined in some parts due to improvement in living conditions globally, Ethiopia is not an exceptional and expansion of major deworming efforts. on the other hand, the increase in population growth in Ethiopia is tremendous and therefore, might have increased the numbers infected and resulted in slight decline in rate. it is also suggested that widespread of monotherapy of antiheminthics for deworming purpose might have facilitated the development of drug resistance and hence, decreased the rate of decline STHs in general and Hookworms in particular $[115,116]$. If environmental and behavioral conditions are not changed at the same time that chemotherapy program is being implemented, the prevalence will tend to return to original pretreatment levels through reinfection and therefore, need holistic approach $[3,116-121]$. According to WHO risk categorization, our finding (33\%) indicating that MRZ of STHs requiring MDA once annually, specifically in SNNPR, Amhara and Oromia regions.

In relation to the species of STH, A. lumbricoides was the predominant species with a prevalence of $17 \%$ indicating that about one in six of Ethiopian children are living with Ascariasis. The current prevalence of the parasite is higher than the findings from other counries such as Iran $(0.75 \%)$ and Srilanka $(2.8 \%)$ which indicated that indoor and outdoor biotic contamination of the living environment arising partly from improper disposal of human waste, and partly from the integration of the lives of humans and animals of Ethiopian community might account for the still high rate of of the infection in the country. The finding of the current review (17\%) is in line with findings from South America (15.6\%), studies conducted in Amhara region, Ethiopia (16.8\%) and the overall burden in Sub-Saharan African countries (15\%) [122]. However, it was lower than results from Nigeria (44.6\%), Rwanda (38.6\%), Uganda (43.5\%) [123], Kenya (24.3\%) [124] and previous estimate in Ethiopia (37\%). The observed differences might be due variation in some factors putting population at risk of acquiring STHs such as geographical variations, life style of the community, soil

Page $15 / 30$ 
humidity and exposure to contaminated environments.. In the current review, the prevalence of $A$. lumbricoides significantly decreased from $25 \%$ in $1997-2012$ to $14 \%$ in $2013-2020(p=0.006)$. There was a $49 \%$ decline in prevalence of $A$. lumbricoides observed before the implementation of MDA program in school children compared to post MDA. This risk reduction might be related to the improved sanitation, access to better water supply, improved personnel hygiene or the higher efficacy of the available treatments against $A$ lumbricoides [115]. In support of this, a local study conducted on the efficacy of albendazole and mebendazole indicated that the drugs have $95 \%$ efficacy in decreasing the burden of the parasite in Ethiopia [125].

The pooled prevalence of $6 \%$ observed for T. trichiura was higher than the $1.9 \%$ and $3.4 \%$ reported from Uganda [112] and Rwanda [114], respectively. The present finding is however, lower than the reports of the disease burden of Sub-saharan Africa (13\%) [106], Nigeria (18.2\%) [110] and Cameroon (15.6\%) [111]. The variations might be due to geographical variations, life style of the community, soil humidity and exposure to contaminated environments. Meta-regression anlaysis by year of publication revealed that the prevalence of $T$. trichiura decreased from $14 \%$ in $1997-2012$ to $4 \%$ in $2013-2020(p=0.03)$. The reason behind the substantial decrease in prevalence of $T$. trichiura in the country during the study period might be due to the synergistic effect of overall improvements in sanitation, personnel hygiene and deworming programmes.

The finding of thee current review showed that the prevalence of hookworms was $12 \%$ indicating that the current finding is lower than others studies conducted in Nigeria (32.7\%) [114] and Uganda (18.5\%) [123]. However, it was higher than studies conducted in Kenya (0.4\%) [124], Rawanda (1.8\%) [126]and Cameroon (3.9\%) [127]. In general, increments of prevalence in our data might be attributed to the re-infection rate, low coverage or unequal distribution of MDA in all regions of the country, level of poverty (walking bare of foot) and lack of good quality of life. For instance, most Ethiopian are living in rural area and engaged in agriculture. Engagement in agricultural pursuits remains a common denominator for adult human hookworm infection,who might be serve as reservoir for reinfection of children [121]. Hookworm did not show significant trend of decrement in prevalence between 1997-2012 (13\%) as compared the years between 2013-2020. This is inconstrast to study conducted in Nepal where the revalence of Hookworms significantly decreased between 1990s to 2015[128].

Eliminating STHs as a public health has to go beyond preventive chemotherapy for school-age children, as other group at risk also serve as reservoir of infection (preschool children and pregnant women and even adults ), which might have resulted in slight decline again. It is also suggested that widespread of monotherapy of antiheminthics for deworming purpose might have facilitated the development of drug resistance and hence, decreased the rate of decline STHs in general and Hookworms in particular $[115,116]$.

The strengths of our review include rigorous search of several databases and other sources to identify eligible studies on the large pediatric population infected by STHs and generate data for policymakers to strengthen or modify the already ongoing control and prevent measures on place. We also estimated the geographical distribution and identified risk areas that should be prioritized for MDA and other control interventions, which complement global efforts towards elimination of STHs and other parasitic infections by 2020. In addition, this work also highlighted the need for survey in areas where data are not available such as Somalia region, Afar region, Harari, Dire Dawa city and Gembela regions or scanty (Addis Ababa city and Benishangul-Gumuz region). There are a few limitations of the present meta-analysis. First, It is prudent to interpret the results of this study as $34(38.6 \%)$ of the included studies were low quality based on our quality assessment criteria. Second, in almost all of the studies included in this review, single stool sample examination were used despite multiple stool samples recommendation for standard diagnosis and therefore, there is possibility for underestimation of the prevalence. Almost all studies included the current analyses examined the stool specimens for many parasites at a time and the diagnostic performance of such an approach is not known compared to studies that examine solely for STHs, such diagnostic approach might affect the detection rate and prevalence estimates of STHs infections. Third WHO has recommended Kato-Katz method as the best and most reliable diagnostic tool with better efficacy, accuracy and predictive value than other techniques in resource poor settings [129]. However, only $39.8 \%$ of the studies reported the use of Kato-Katz method or in combination with other methods. Morbidity due to STH infections is a result of worm burden (number of eggs per gram of feces), otherwise called infection intesity. The disease prevalence is commonly combined with the intensity of infection to assess the epidemiological situation for STH infections and to classify communities into transmission categories, which enables the determination of the appropriate strategies for treatment and control [130]. However, only few studies (13 out of 88) reported the intensity of infection of species-specific STHs and thus, difficult to reach on definitive conclusion about intensity of infection of STH in Ethiopia children. Therefore, there is urgent need of large-scale study to assess the inetsnity of infection of STH in children using sensitive diagnostic tool on repeated stool sample. Finally, the review protocol was not registered a head of actual meta-analysis, which could be source of bias.

Page $16 / 30$ 


\section{Conclusions}

Despite efforts made to reduce, STHs infection is still highly prevalent across Ethiopian region with some degree of variation. Southern, Amhara and Oromia regional states carry the highest burden. We observed a decreased prevalence of STHs among Ethiopian children post MDA compared to preMDA, but the decrease is not statistically significant. A. lumbricoides had the highest prevalence among STHs. A. lumbricoides and T. trichiura were the most prevalent species in the Southern region while, hookworms recorded the highest prevalence in Amhara region. With effort made by the country in eradicating STHs infections, none of the regions in the country is classified as HRZ according to WHO risk classification. Southern, Amhara and Oromia regions carried moderate burden and are classified as MRZs and therefore annual MDA is recommended while, the rest of the regions and city are classified as low risk zones LRZs. We hope that the findings of the current study provide valuable information to the policymakers, National Health Bureau and other concerned bodies, specifically on endemicity of STHs, national and regional distribution and their prevalence and species distribution in Ethiopia.

\section{Lists Of Abbreviations}

STH, soil transmitted helminth; MRZ, moderate risk zone; HRZ, high risk zone; LRZ, low risk zone; MDA, mass drug administration; GRADE, Grading of Recommendations Assessment, Development and Evaluation; Cl, confidence interval; PRISMA, Preferred Reporting Items for Systematic Reviews and Meta-Analyses; WHO, world health organization; NTDs, Neglected tropical diseases.

\section{Declarations}

\section{Ethics approval and consent to participate:}

None applicable

\section{Consent for publication:}

Not applicable.

\section{Availability of data and materials:}

The datasets used and/or analyzed during the current study are available from the corresponding author on reasonable request

\section{Competing interests:}

The authors declare that they have no competing interests.

\section{Funding:}

we did not receive any funding support for this work

\section{Authors' contributions:}

LCH and TM conceived the study. LCH and TM extracted the data, and independently decided for inclusion or exclusion, and in events of disagreement, WJ helped to resolve. LCH and DE performed all the statistical analyses. LCH and WJ prepared manuscript with the help from DE. All authors read and approved the final manuscript.

\section{Acknowledgements:}

None

\section{Authors' information:}

Department of Clinical Pharmacy, School of Pharmacy, College Health Sciences, Jimma University, Jimma, Ethiopia Legese Chelkeba (PhD, Associate Professor of Clinical pharmacy) 
Tsegaye Melaku (MSc, Assiatnt profeesor of Clinical pharmacy)

Department of Parasitology and Molecular biology, School of Medical Laboratory Sciences, College Health Sciences, Jimma University, Jimma, Ethiopia

Daniel Emana (MSc, PhD student)

Worku Jimma (PhD, Assistant professor of Health information management)

Department of information Sciences, College of Natural Sciences, Jimma University, Jimma, Ethiopia.

\section{References}

1. Savioli L, et al. Schistosomiasis and soil-transmitted helminth infections: forging control efforts. Trans R Soc Trop Med Hyg. 2002;96(6):577.

2. Helminthiases WS-t. Eliminating soil-transmitted helminthiases as a public health problem in children: progress report 20012010 and strategic plan 2011-2020. France: World Health Organization; 2012. pp. 19-29.

3. Bethony J, et al. Soil-transmitted helminth infections: ascariasis, trichuriasis, and hookworm. The lancet. 2006;367(9521):152132.

4. Gyorkos TW, et al. Trichuris and hookworm infections associated with anaemia during pregnancy. Tropical Med Int Health. $2011 ; 16(4): 531-7$.

5. Hotez PJ, et al. Helminth infections: the great neglected tropical diseases. J Clin Investig. 2008;118(4):1311-21.

6. Mekonnen Z, et al. Comparison of individual and pooled stool samples for the assessment of soil-transmitted helminth infection intensity and drug efficacy. PLoS Negl Trop Dis. 2013;7(5):e2189.

7. Tefera T, et al., Parasitic contamination of fruits and vegetables collected from selected local markets of Jimma Town, Southwest Ethiopia. International scholarly research notices, 2014. 2014.

8. Organization WH. Prevention and control of schistosomiasis and soil-transmitted helminthiasis: report of a WHO expert committee. 2002.

9. Pullan RL, et al. Global numbers of infection and disease burden of soil transmitted helminth infections in 2010. Parasites vectors. $2014 ; 7(1): 37$.

10. Östan İ, et al. Health inequities: lower socio-economic conditions and higher incidences of intestinal parasites. BMC Public Health. $2007 ; 7(1): 342$.

11. De Silva NR, et al. Soil-transmitted helminth infections: updating the global picture. Trends Parasitol. 2003;19(12):547-51.

12. Organization WH, Accelerating work to overcome the global impact of neglected tropical diseases: a roadmap for implementation: executive summary. 2012, World Health Organization.

13. Silver ZA, et al. Geographical distribution of soil transmitted helminths and the effects of community type in South Asia and South East Asia-A systematic review. PLoS Negl Trop Dis. 2018;12(1):e0006153.

14. Nute AW, et al., Prevalence of soil-transmitted helminths and Schistosoma mansoni among a population-based sample of schoolage children in Amhara region, Ethiopia. Parasites \& vectors, 2018. 11(1): p. 431.

15. Mekonnen Z, et al., Assessment of seasonality in soil-transmitted helminth infections across 14 schools in Jimma Town, Ethiopia. The Pan African Medical Journal, 2019. 32.

16. Gadisa E, Jote K. Prevalence and factors associated with intestinal parasitic infection among under-five children in and around Haro Dumal Town, Bale Zone, Ethiopia. BMC Pediatr. 2019;19(1):385.

17. Moher D, et al. Preferred reporting items for systematic reviews and meta-analyses: the PRISMA statement. Ann Intern Med. 2009;151(4):264-9.

18. Atkins D, et al. Systems for grading the quality of evidence and the strength of recommendations I: critical appraisal of existing approaches The GRADE Working Group. BMC Health Serv Res. 2004;4(1):38.

19. Alamir M, Awoke W, Feleke A. Intestinal parasites infection and associated factors among school children in Dagi primary school. Amhara National Regional State Ethiopia Health. 2013;5(10):1697.

Page 18/30 
20. Demissie F, Petros B, Kebede A. Hookworm species distribution among school children in Asendabo town, Jimma Zone, South West Ethiopia. Ethiopian Journal of Health Sciences, 2008. 18(2).

21. Desalegn A, Mossie A, Gedefaw L. Nutritional iron deficiency anemia: magnitude and its predictors among school age children, southwest Ethiopia: a community based cross-sectional study. PloS one. 2014;9(12):e114059.

22. Fentie T, et al. Epidemiology of human fascioliasis and intestinal parasitosis among schoolchildren in Lake Tana Basin, northwest Ethiopia. Trans R Soc Trop Med Hyg. 2013;107(8):480-6.

23. Haileamlak A. Intestinal parasites in asymptotic children in Southwest Ethiopia. Ethiopian Journal of Health Sciences, 2005. 15(2).

24. Mulatu G, et al., Intestinal parasitic infections among children under five years of age presenting with diarrhoeal diseases to two public health facilities in Hawassa, South Ethiopia. Infectious diseases of poverty, 2015. 4(1): p. 49.

25. Alemu A, et al. Soil transmitted helminths and Schistosoma mansoni infections among school children in Zarima town, northwest Ethiopia. BMC Infect Dis. 2011;11(1):189.

26. Amare B, et al. Nutritional status, intestinal parasite infection and allergy among school children in Northwest Ethiopia. BMC Pediatr. 2013;13(1):7.

27. Ayalew A, Debebe T, Worku A. Prevalence and risk factors of intestinal parasites among Delgi school children, North Gondar, Ethiopia. Journal of Parasitology Vector Biology. 2011;3(5):75-81.

28. Beyene G, Tasew H. Prevalence of intestinal parasite, Shigella and Salmonella species among diarrheal children in Jimma health center, Jimma southwest Ethiopia: a cross sectional study. Ann Clin Microbiol Antimicrob. 2014;13(1):10.

29. Legesse M, Erko B. Prevalence of intestinal parasites among schoolchildren in a rural area close to the southeast of Lake Langano, Ethiopia. Ethiop J Health Dev. 2004;18:116. ., 120.

30. Merid Y, et al. Intestinal helminthic infection among children at Lade Awassa Area, South Ethiopia. Ethiopian Journal of Health Development. 2001;15(1):31-8.

31. Nguyen NL, et al. Intestinal parasitic infection and nutritional status among school children in Angolela, Ethiopia. J Prev Med Hyg. 2012;53(3):157.

32. Nyantekyi LA, et al., Intestinal parasitic infections among under-five children and maternal awareness about the infections in Shesha Kekele, Wondo Genet, Southern Ethiopia. Ethiopian Journal of Health Development, 2010. 24(3).

33. Tadesse G. The prevalence of intestinal helminthic infections and associated risk factors among school children in Babile town, eastern Ethiopia. Ethiopian Journal of Health Development. 2005;19(2):140-7.

34. Degarege A, Erko B. Association between intestinal helminth infections and underweight among school children in Tikur Wuha Elementary School, Northwestern Ethiopia. J Infect Public Health. 2013;6(2):125-33.

35. Jemaneh L. Schistosomiasis mansoni and geo-Helminthiasis in school children in the Dembia Plains, Northwest Ethiopia. The Ethiopian Journal of Health Development (EJHD), 1998. 12(3).

36. Jejaw A, et al. High prevalence of Schistosoma mansoni and other intestinal parasites among elementary school children in Southwest Ethiopia: a cross-sectional study. BMC Public Health. 2015;15(1):600.

37. King JD, et al. Intestinal parasite prevalence in an area of Ethiopia after implementing the SAFE strategy, enhanced outreach services, and health extension program. PLoS Negl Trop Dis. 2013;7(6):e2223.

38. Tulu B, Taye S, Amsalu E. Prevalence and its associated risk factors of intestinal parasitic infections among Yadot primary school children of South Eastern Ethiopia: a cross-sectional study. BMC Res Notes. 2014;7(1):848.

39. Workneh T, Esmael A, Ayichiluhm M. Prevalence of intestinal parasitic infections and associated factors among Debre Elias primary schools children, East Gojjam Zone, Amhara Region, North West Ethiopia. J Bacteriol Parasitol. 2014;15(1):1-5.

40. Erosie L, et al. Prevalence of Hookworm infection and haemoglobin status among rural elementary school children in Southern Ethiopia. Ethiopian Journal of Health Development. 2002;16(1):113-5.

41. Gelaw A, et al. Prevalence of intestinal parasitic infections and risk factors among schoolchildren at the University of Gondar Community School, Northwest Ethiopia: a cross-sectional study. BMC Public Health. 2013;13(1):304.

42. Jemaneh L. Soil-transmitted helminth infections and Schistosomiasis mansoni in school children from Chilga District, Northwest Ethiopia. Ethiopian journal of health sciences, 2001. 11(2).

Page 19/30 
43. Mahmud MA, et al. Risk factors for intestinal parasitosis, anaemia, and malnutrition among school children in Ethiopia. Pathogens global health. 2013;107(2):58-65.

44. Mahmud MA, et al. Efficacy of handwashing with soap and nail clipping on intestinal parasitic infections in school-aged children: a factorial cluster randomized controlled trial. PLoS Med. 2015;12(6):e1001837.

45. Reji P, et al., Intestinal parasitic infections and malnutrition amongst first-cycle primary schoolchildren in Adama, Ethiopia. African journal of primary health care \& family medicine, 2011. 3(1).

46. Roma B, Worku S. Magnitude of Schistosoma mansoni and intestinal helminthic infections among school children in WondoGenet Zuria, southern Ethiopa. Ethiopian Journal of Health Development. 1997;11:125-30.

47. Wegayehu T, Adamu H, Petros B. Prevalence of Giardia duodenalis and Cryptosporidium species infections among children and cattle in North Shewa Zone, Ethiopia. BMC Infect Dis. 2013;13(1):419.

48. Adamu $\mathrm{H}$, et al. The prevalence of intestinal parasites in paediatric diarrhoeal and non-diarrhoeal patients in Addis Ababa hospitals, with special emphasis on opportunistic parasitic infections and with insight into the demographic and socio-economic factors. Ethiopian Journal of Health Development. 2006;20(1):39-46.

49. Belyhun Y, et al. Prevalence and risk factors for soil-transmitted helminth infection in mothers and their infants in Butajira, Ethiopia: a population based study. BMC Public Health. 2010;10(1):21.

50. Dejenie T, Asmelash T, Teferi M. Intestinal helminthes infections and re-infections with special emphasis on schistosomiasis mansoni in Waja, North Ethiopia. Momona Ethiopian Journal of Science, 2009. 1(2).

51. Kidane E, et al. Prevalence of intestinal parasitic infections and their associations with anthropometric measurements of school children in selected primary schools, Wukro town, eastern Tigray, Ethiopia. Scientific Journal of Zoology. 2013;229(1319):1-16.

52. Legesse L, Erko B, Hailu A. Current status of intestinal Schistosomiasis and soiltransmitted helminthiasis among primary school children in Adwa Town, Northern Ethiopia. Ethiopian Journal of Health Development, 2010. 24(3).

53. Mathewos B, et al. Current status of soil transmitted helminths and Schistosoma mansoni infection among children in two primary schools in North Gondar, Northwest Ethiopia: a cross sectional study. BMC Research Notes. 2014;7(1):88.

54. Alemayehu B, et al. Epidemiology of intestinal helminthiasis among school children with emphasis on Schistosoma mansoni infection in Wolaita zone, Southern Ethiopia. BMC Public Health. 2017;17(1):587.

55. Assefa A, Dejenie T, Tomass Z. Infection prevalence of Schistosoma mansoni and associated risk factors among schoolchildren in suburbs of Mekelle city, Tigray, Northern Ethiopia. Momona Ethiopian Journal of Science. 2013;5(1):174-88.

56. Dejenie T, Petros B. Irrigation practices and intestinal helminth infections in southern and central zones of Tigray. Ethiopian Journal of Health Development, 2009. 23(1).

57. Gashaw F, et al., Prevalence of intestinal helminth infection among school children in Maksegnit and Enfranz Towns, northwestern Ethiopia, with emphasis on Schistosoma mansoni infection. Parasites \& vectors, 2015. 8(1): p. 567.

58. Jemaneh L. Intestinal helminth infections in school children in Adarkay District, Northwest Ethiopia, with special reference to schistosoma mansoni. Ethiop J Health Dev. 1997;11(3):289-94.

59. Mengist HM, Taye B, Tsegaye A. Intestinal parasitosis in relation to CD4 + T cells levels and anemia among HAART initiated and HAART naive pediatric HIV patients in a model ART center in Addis Ababa, Ethiopia. PloS one. 2015;10(2):e0117715.

60. Tekeste Z, et al. Epidemiology of intestinal schistosomiasis and soil transmitted helminthiasis among primary school children in Gorgora, Northwest Ethiopia. Asian Pacific Journal of Tropical Disease. 2013;3(1):61-4.

61. Terefe A, et al. Schistosomiasis mansoni and soil-transmitted helminthiasis in Bushulo village, southern Ethiopia. Ethiopian Journal of Health Development. 2011;25(1):46-50.

62. Wale $M$, Wale $M$, Fekensa $T$. The prevalence of intestinal helminthic infections and associated risk factors among school children in Lumame town, Northwest, Ethiopia. Journal of Parasitology Vector Biology. 2014;6(10):156-65.

63. Aiemjoy K, et al. Epidemiology of soil-transmitted helminth and intestinal protozoan infections in preschool-aged children in the Amhara region of Ethiopia. Am J Trop Med Hyg. 2017;96(4):866-72.

64. Alemu M, Hailu A, Bugssa G. Prevalence of intestinal schistosomiasis and soil-transmitted helminthiasis among primary schoolchildren in Umolante district, South Ethiopia. Clin Med Res. 2014;3(6):174-80.

65. Begna T, Solomon T, Yohannes EA. ZENEBE, Intestinal parasitic infections and nutritional status among primary school children in Delo-mena district, South Eastern Ethiopia. Iranian journal of parasitology. 2016;11(4):549. 
66. Dejenie T, Asmelash T. Schistosomiasis mansoni among school children of different water source users in Tigray, Northern Ethiopia. Momona Ethiopian Journal of Science, 2010. 2(1).

67. Teklemariam A, Dejenie T, Tomass Z. Infection prevalence of intestinal helminths and associated risk factors among schoolchildren in selected kebeles of Enderta district, Tigray, Northern Ethiopia. Journal of Parasitology Vector Biology. 2014;6(11):166-73.

68. Zemene T, Shiferaw MB, Prevalence of intestinal parasitic infections in children under the age of 5 years attending the Debre Birhan referral hospital, North Shoa, Ethiopia. BMC research notes, 2018. 11(1): p. 58.

69. Firdu T, Abunna F, Girma M, Intestinal protozoal parasites in diarrheal children and associated risk factors at Yirgalem Hospital, Ethiopia: A case-control study. International scholarly research notices, 2014. 2014.

70. Tefera E, et al., Prevalence and intensity of soil transmitted helminths among school children of Mendera Elementary School, Jimma, Southwest Ethiopia. The Pan African Medical Journal, 2017. 27.

71. Unasho A. An investigation of intestinal parasitic infections among the asymptomatic children in, Southern Ethiopia. International Journal of Child Health Nutrition. 2013;2(3):212-22.

72. Yimam Y, Degarege A, Erko B. Effect of anthelminthic treatment on helminth infection and related anaemia among school-age children in northwestern Ethiopia. BMC Infect Dis. 2016;16(1):613.

73. Abdi M, Nibret E, Munshea A. Prevalence of intestinal helminthic infections and malnutrition among schoolchildren of the Zegie Peninsula, northwestern Ethiopia. J Infect Public Health. 2017;10(1):84-92.

74. Abera A, Nibret E. Prevalence of gastrointestinal helminthic infections and associated risk factors among schoolchildren in Tilili town, northwest Ethiopia. Asian Pacific journal of tropical medicine. 2014;7(7):525-30.

75. Wegayehu T, et al. Multilocus genotyping of Giardia duodenalis isolates from children in Oromia Special Zone, central Ethiopia. BMC microbiology. 2016;16(1):89.

76. Abossie A, Seid M. Assessment of the prevalence of intestinal parasitosis and associated risk factors among primary school children in Chencha town, Southern Ethiopia. BMC Public Health. 2014;14(1):166.

77. Hailegebriel T. Prevalence of intestinal parasitic infections and associated risk factors among students at Dona Berber primary school, Bahir Dar, Ethiopia. BMC Infect Dis. 2017;17(1):362.

78. Alemu G, Aschalew Z, Zerihun E. Burden of intestinal helminths and associated factors three years after initiation of mass drug administration in Arbaminch Zuria district, southern Ethiopia. BMC Infect Dis. 2018;18(1):435.

79. Alemu A, et al. Schistosoma mansoni and soil-transmitted helminths among preschool-aged children in Chuahit, Dembia district, Northwest Ethiopia: prevalence, intensity of infection and associated risk factors. BMC Public Health. 2016;16(1):422.

80. Alemu G, Abossie A, Yohannes Z. Current status of intestinal parasitic infections and associated factors among primary school children in Birbir town, Southern Ethiopia. BMC Infect Dis. 2019;19(1):270.

81. Bajiro M, et al., Prevalence of Schistosoma mansoni infection and the therapeutic efficacy of praziquantel among school children in Manna District, Jimma Zone, southwest Ethiopia. Parasites \& vectors, 2016. 9(1): p. 560.

82. Amor A, et al. High prevalence of Strongyloides stercoralis in school-aged children in a rural highland of north-western Ethiopia: the role of intensive diagnostic work-up. Parasites vectors. 2016;9(1):617.

83. Gebretsadik D, et al. Prevalence of intestinal parasitic infection among children under 5 years of age at Dessie Referral Hospital: cross sectional study. BMC Res Notes. 2018;11(1):771.

84. Bekana T, et al. Transmission of Schistosoma mansoni in Yachi areas, southwestern Ethiopia: new foci. Infectious diseases of poverty. 2019;8(1):1.

85. Diro E, et al. Clinical aspects of paediatric visceral leishmaniasis in N orth-west E thiopia. Tropical Med Int Health. 2015;20(1):816.

86. Birhanu M, Gedefaw L, Asres Y. Anemia among School-Age Children: Magnitude, Severity and Associated Factors in Pawe Town, Benishangul-Gumuz Region, Northwest Ethiopia. Ethiopian journal of health sciences. 2018;28(3):259-66.

87. Leta GT, et al. Comparison of individual and pooled diagnostic examination strategies during the national mapping of soiltransmitted helminths and Schistosoma mansoni in Ethiopia. PLoS Negl Trop Dis. 2018;12(9):e0006723.

88. Tefera E, Mohammed J, Mitiku H. Intestinal helminthic infections among elementary students of Babile town, eastern Ethiopia. Pan African Medical Journal, 2015. 20(1).

Page $21 / 30$ 
89. Hailu T, et al. Multivariate analysis of factors associated with Schistosoma mansoni and hookworm infection among primary school children in rural Bahir Dar. Northwest Ethiopia. Tropical diseases, travel medicine vaccines. 2018;4(1):4.

90. Alemayehu B, Tomass Z. Schistosoma mansoni infection prevalence and associated risk factors among schoolchildren in Demba Girara, Damot Woide District of Wolaita Zone, Southern Ethiopia. Asian Pacific journal of tropical medicine. 2015;8(6):457-63.

91. Ali I, Mekete G, Wodajo N. Intestinal parasitism and related risk factors among students of Asendabo Elementary and Junior Secondary School South western Ethiopia. Ethiopian Journal of Health Development. 1999;13(2):157-62.

92. Jemaneh L, Intestinal helminth infections in schoolchildren in Gonder town and surrounding areas, Northwest Ethiopia. SINET: Ethiopian Journal of Science, 1999. 22(2): p. 209-220.

93. Debalke S, et al. Soil transmitted helminths and associated factors among schoolchildren in government and private primary school in Jimma Town, Southwest Ethiopia. Ethiopian journal of health sciences. 2013;23(3):237-44.

94. Dejene T. Impact of irrigation on the prevalence of intestinal parasite infections with emphasis on schistosomiasis in HintalloWejerat, North Ethiopia. Ethiopian Journal of Health Sciences, 2008. 18(2).

95. Abera B, et al. Epidemiology of soil-transmitted helminths, Schistosoma mansoni, and haematocrit values among schoolchildren in Ethiopia. The Journal of Infection in Developing Countries. 2013;7(03):253-60.

96. Kabeta A, et al. Intestinal parasitic infections and nutritional status of pre-school children in Hawassa Zuria District, South Ethiopia. African Journal of Microbiology Research. 2017;11(31):1243-51.

97. Shumbej T, et al. Soil-transmitted helminths and associated factors among pre-school children in Butajira town, south-Central Ethiopia: a community-based cross-sectional study. PloS one. 2015;10(8):e0136342.

98. Tadege B, Shimelis T. Infections with Schistosoma mansoni and geohelminths among school children dwelling along the shore of the Lake Hawassa, southern Ethiopia. PloS one. 2017;12(7):e0181547.

99. Asemahagn MA. Parasitic infection and associated factors among the primary school children in Motta town, western Amhara, Ethiopia. American Journal of Public Health Research. 2014;2(6):248-54.

100. Teshale T, et al., Prevalence of intestinal helminths and associated factors among school children of Medebay Zana wereda; North Western Tigray, Ethiopia 2017. BMC research notes, 2018. 11(1): p. 444.

101. FELEKE BE. Epidemiology of Hookworm Infection in the School-age Children: A Comparative Cross-sectional Study. Iranian journal of parasitology. 2018;13(4):560.

102. Eyamo T, et al., Soil-Transmitted Helminths And Other Intestinal Parasites Among Schoolchildren In Southern Ethiopia. Research and reports in tropical medicine, 2019. 10: p. 137.

103. Mekonnen Z, et al. Intestinal polyparasitism with special emphasis to soil-transmitted helminths among residents around Gilgel Gibe Dam, Southwest Ethiopia: a community based survey. BMC Public Health. 2016;16(1):1185.

104. Molla E, Mamo H. Soil-transmitted helminth infections, anemia and undernutrition among schoolchildren in Yirgacheffee, South Ethiopia. BMC Res Notes. 2018;11(1):585.

105. Sitotaw B, Mekuriaw H, Damtie D. Prevalence of intestinal parasitic infections and associated risk factors among Jawi primary school children, Jawi town, north-west Ethiopia. BMC Infect Dis. 2019;19(1):341.

106. Tadesse M, Dobo B, Birmeka M. Prevalence and associated risk factors of intestinal parasitic infections among school children in Bamo no. 2 primary school, Adele town, East Arsi, Ethiopia. Sub-Saharan African Journal of Medicine. 2019;6(2):77.

107. Weldesenbet $\mathrm{H}$, Worku A, Shumbej T. Prevalence, infection intensity and associated factors of soil transmitted helminths among primary school children in Gurage zone, South Central Ethiopia: a cross-sectional study design. BMC Res Notes. 2019;12(1):231.

108. Workineh L, et al., Prevalence of Soil-Transmitted Helminth and Schistosoma mansoni Infection and Their Associated Factors among Hiruy Abaregawi Primary School Children, Rural Debre Tabor, North West Ethiopia: A Cross-Sectional Study. Journal of Parasitology Research, 2020. 2020.

109. Shumbej T, Girum T. Helminth infections in light of an ongoing intervention in endemic areas of Guragae zone, Southern Ethiopia: an implication for neglected tropical diseases elimination in Ethiopia by 2020. Tropical diseases, travel medicine and vaccines, 2019. 5(1): p. 8.

110. Siraj S, Alemayehu E, Woldemichael K. Prevalence of Intestinal Parasitic Infections and Associated Factors Among Street Children in Jimma Town; South West Ethiopia in 2019: A Cross Sectional Study. 2019.

Page 22/30 
111. Chammartin F, et al. Soil-transmitted helminth infection in South America: a systematic review and geostatistical meta-analysis. The Lancet infectious diseases. 2013;13(6):507-18.

112. Daryani A, et al. Intestinal parasitic infections in Iranian preschool and school children: A systematic review and meta-analysis. Acta Trop. 2017;169:69-83.

113. Yapi RB, et al., Bayesian risk profiling of soil-transmitted helminth infections and estimates of preventive chemotherapy for school-aged children in Côte d'Ivoire. Parasites \& vectors, 2016. 9(1): p. 162.

114. Karshima SN. Prevalence and distribution of soil-transmitted helminth infections in Nigerian children: a systematic review and meta-analysis. Infectious diseases of poverty. 2018;7(1):69.

115. Keiser J, Utzinger J. Efficacy of current drugs against soil-transmitted helminth infections: systematic review and meta-analysis. Jama. 2008;299(16):1937-48.

116. Levecke B, et al., Assessment of anthelmintic efficacy of mebendazole in school children in six countries where soil-transmitted helminths are endemic. PLoS neglected tropical diseases, 2014. 8(10).

117. Asaolu S, Ofoezie I. The role of health education and sanitation in the control of helminth infections. Acta Trop. 2003;86(23):283-94.

118. Barker WH, Perspectives on acute enteric disease epidemiology and control. Bulletin of the Pan American Health Organization (PAHO); 9 (2), 1975, 1975.

119. Okun DA. The value of water supply and sanitation in development: an assessment. American journal of public health. 1988;78(11):1463-7.

120. Prichard RK, et al., A research agenda for helminth diseases of humans: intervention for control and elimination. PLoS neglected tropical diseases, 2012. 6(4).

121. Hotez PJ, Pritchard DI. Hookworm infection. Sci Am. 1995;272(6):68-74.

122. Hotez PJ, Kamath A. Neglected tropical diseases in sub-Saharan Africa: review of their prevalence, distribution, and disease burden. PLoS neglected tropical diseases, 2009. 3(8).

123. Ojja S, et al. Prevalence, intensity and factors associated with soil-transmitted helminths infections among preschool-age children in Hoima district, rural western Uganda. BMC Infect Dis. 2018;18(1):408.

124. Ngonjo T, et al., Current status of soil-transmitted helminths among school children in kakamega county, western kenya. Journal of parasitology research, 2016. 2016.

125. Tefera E, et al., Therapeutic efficacy of different brands of albendazole against soil transmitted helminths among students of Mendera Elementary School, Jimma, Southwest Ethiopia. Pan African Medical Journal, 2015. 22(1).

126. Staudacher $\mathrm{O}$, et al. Soil-transmitted helminths in southern highland $\mathrm{R}$ wanda: associated factors and effectiveness of schoolbased preventive chemotherapy. Tropical Med Int Health. 2014;19(7):812-24.

127. Tchuenté L-AT, et al. Mapping of schistosomiasis and soil-transmitted helminthiasis in the regions of centre, east and west Cameroon. PLoS Negl Trop Dis. 2012;6(3):e1553.

128. Kunwar R, Acharya L, Karki S. Trends in prevalence of soil-transmitted helminth and major intestinal protozoan infections among school-aged children in Nepal. Tropical Med Int Health. 2016;21(6):703-19.

129. Nikolay B, Brooker SJ, Pullan RL. Sensitivity of diagnostic tests for human soil-transmitted helminth infections: a meta-analysis in the absence of a true gold standard. International journal for parasitology. 2014;44(11):765-74.

130. Organization WH, Preventive chemotherapy in human helminthiasis. Coordinated use of anthelminthic drugs in control interventions: a manual for health professionals and programme managers. 2006: World Health Organization.

\section{Figures}




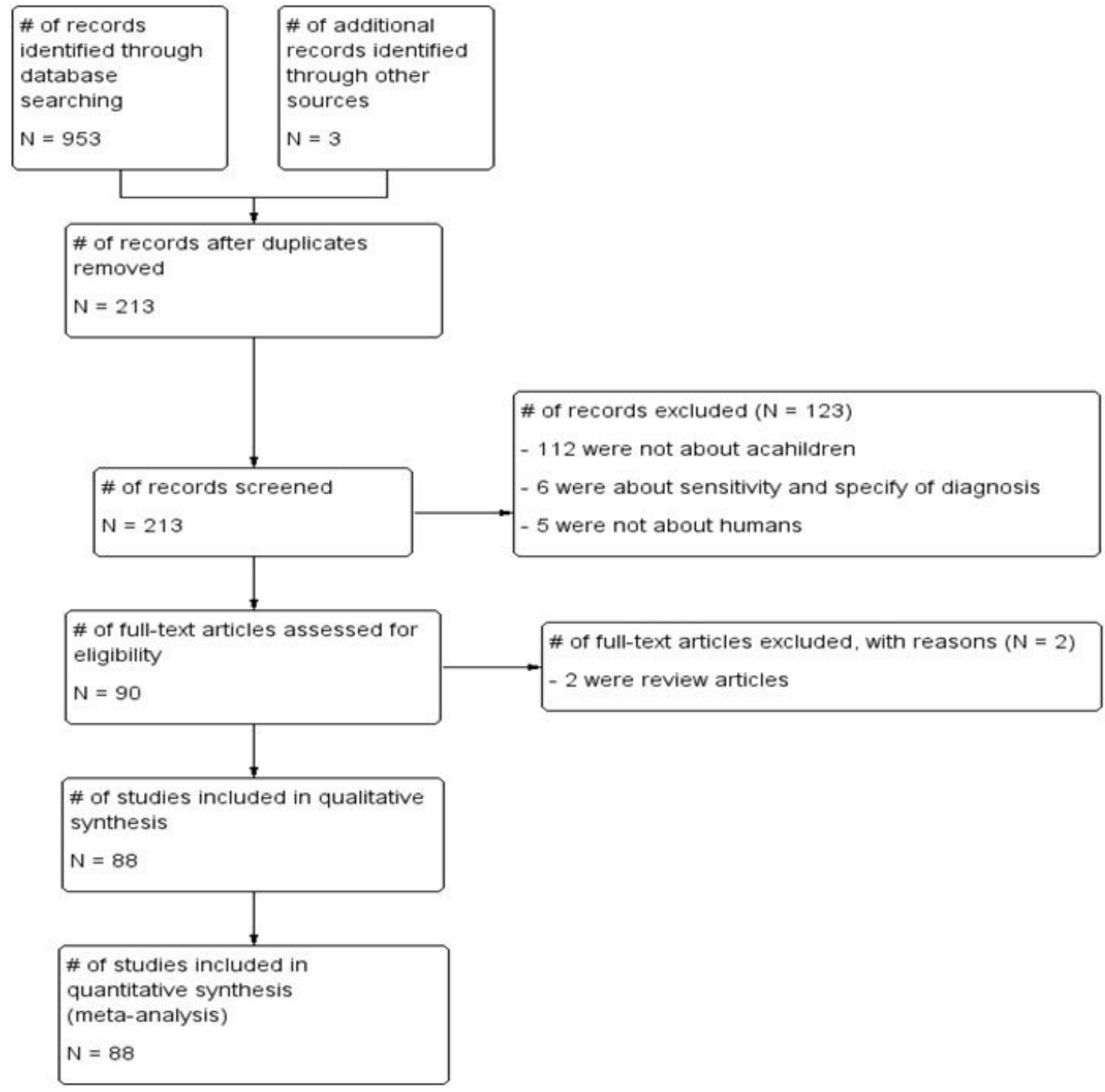

\section{Figure 1}

Flow diagram showing the selection process 


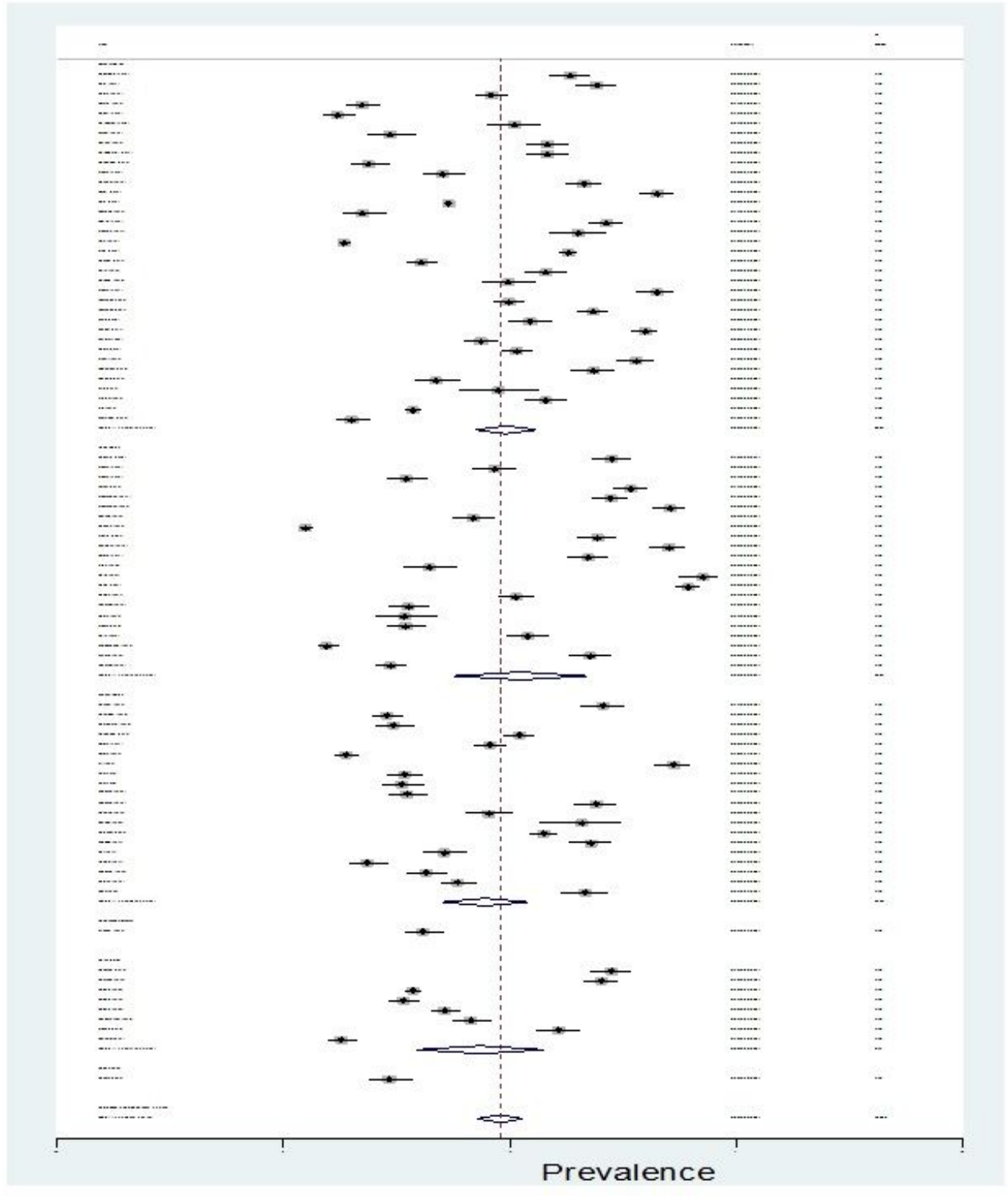

\section{Figure 2}

Forest plot showing pooled prevalence of intestinal parasites among children according to regional states in Ethiopia 


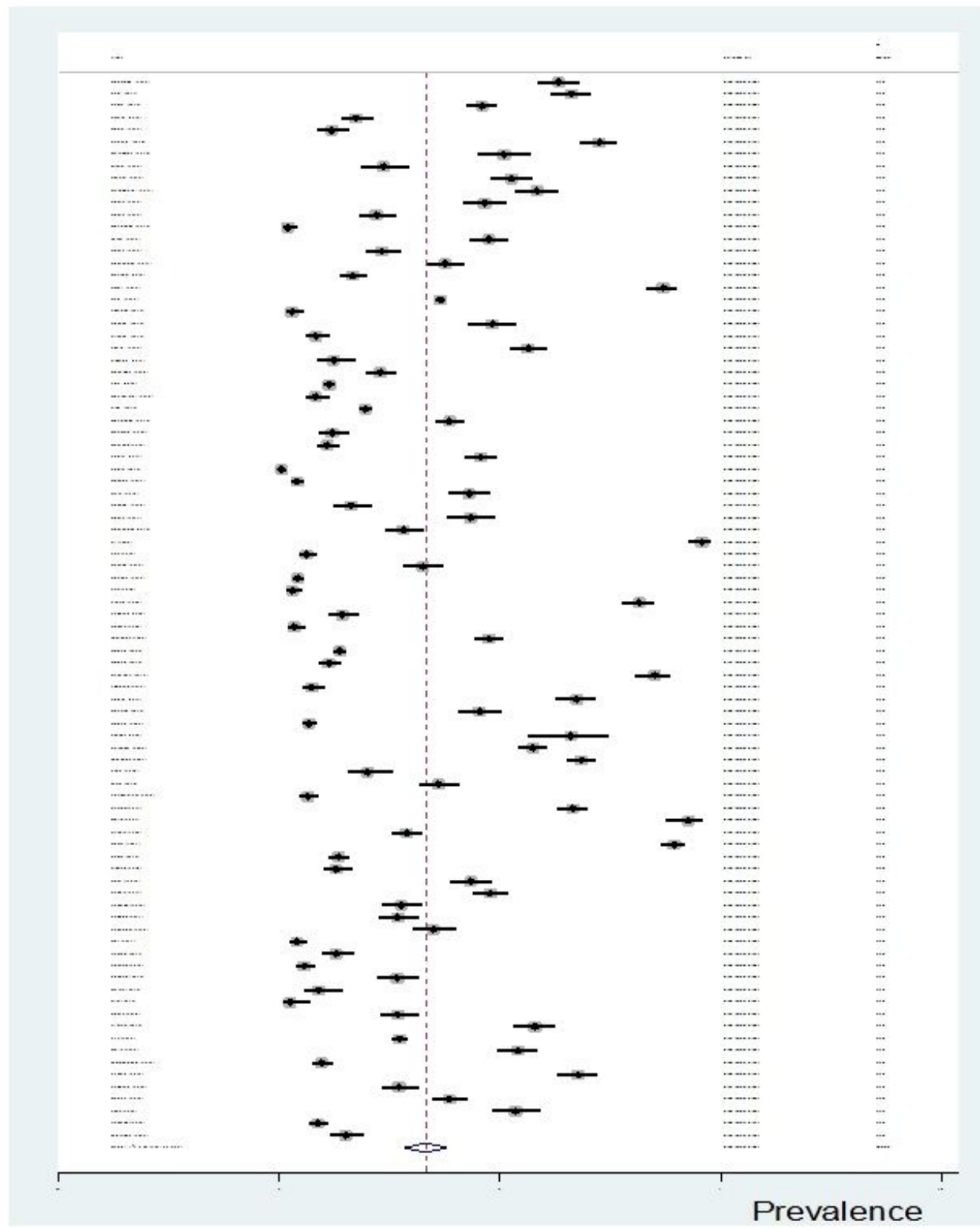

Figure 3

Forest plot showing pooled prevalence of STHs 


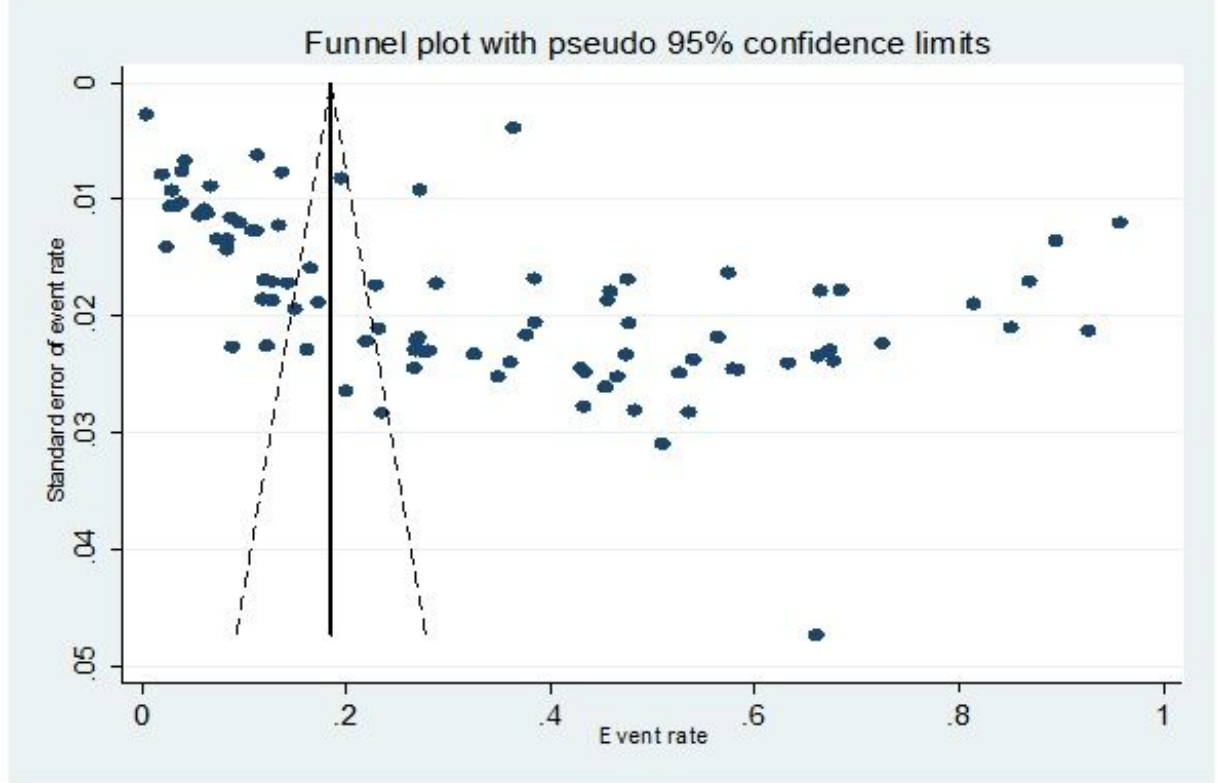

\section{Figure 4}

Publication bias assessment plot: Egger's regression test $(p<0.0001)$ and Begg's rank correlation $(p<0.001)$

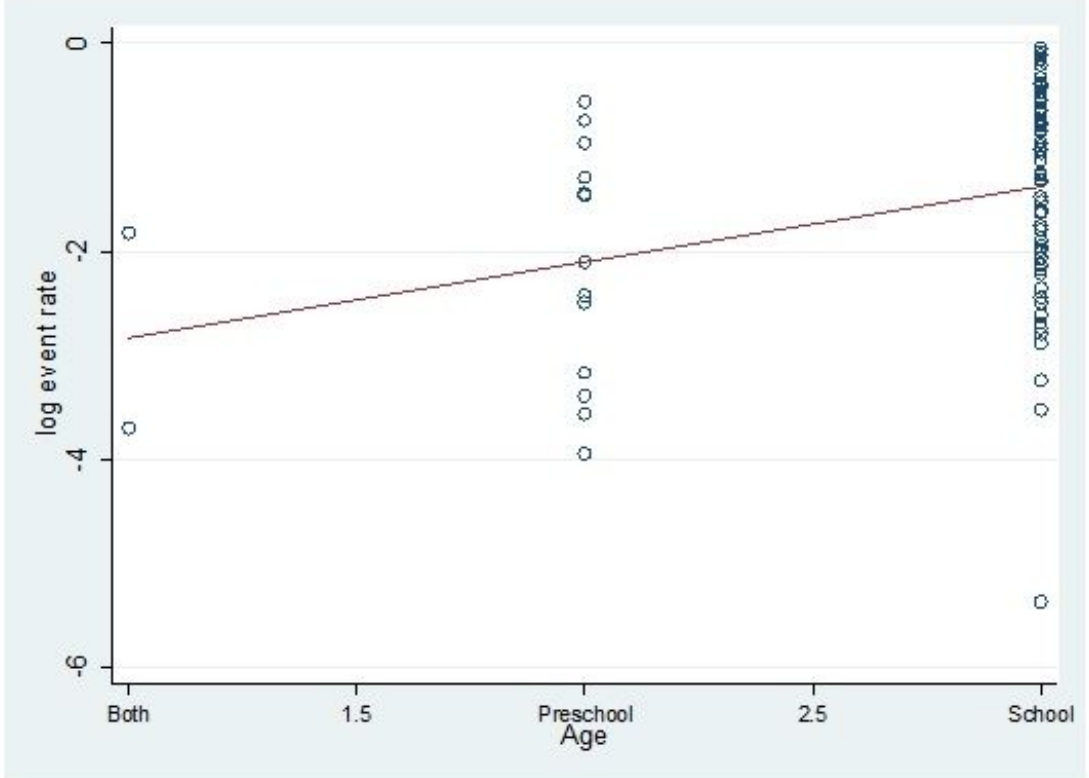

\section{Figure 5}

Meta-regression of prevalence of STHs (log event rate) by Age 


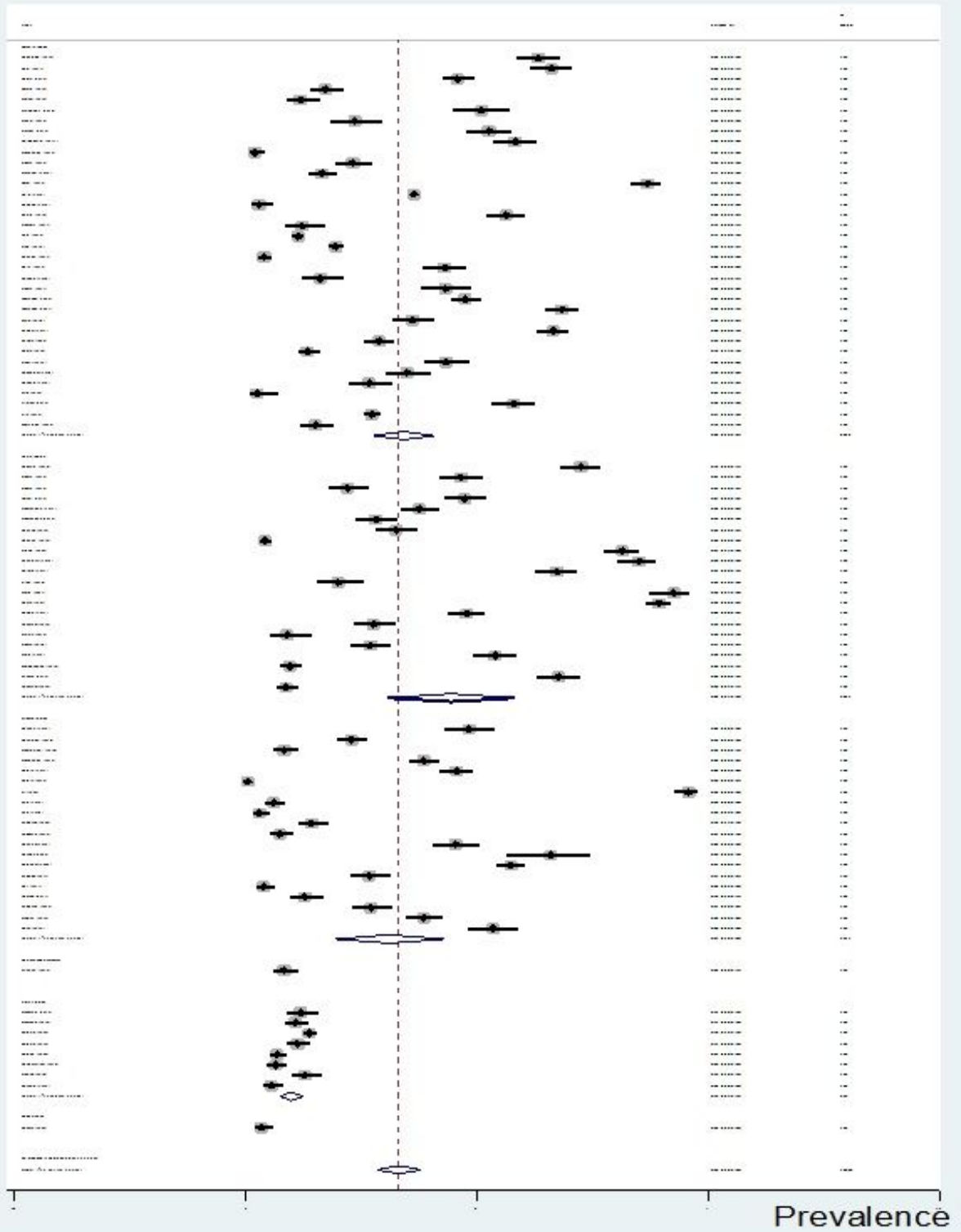

Figure 6

Forest plot showing prevalence of STHs by region 


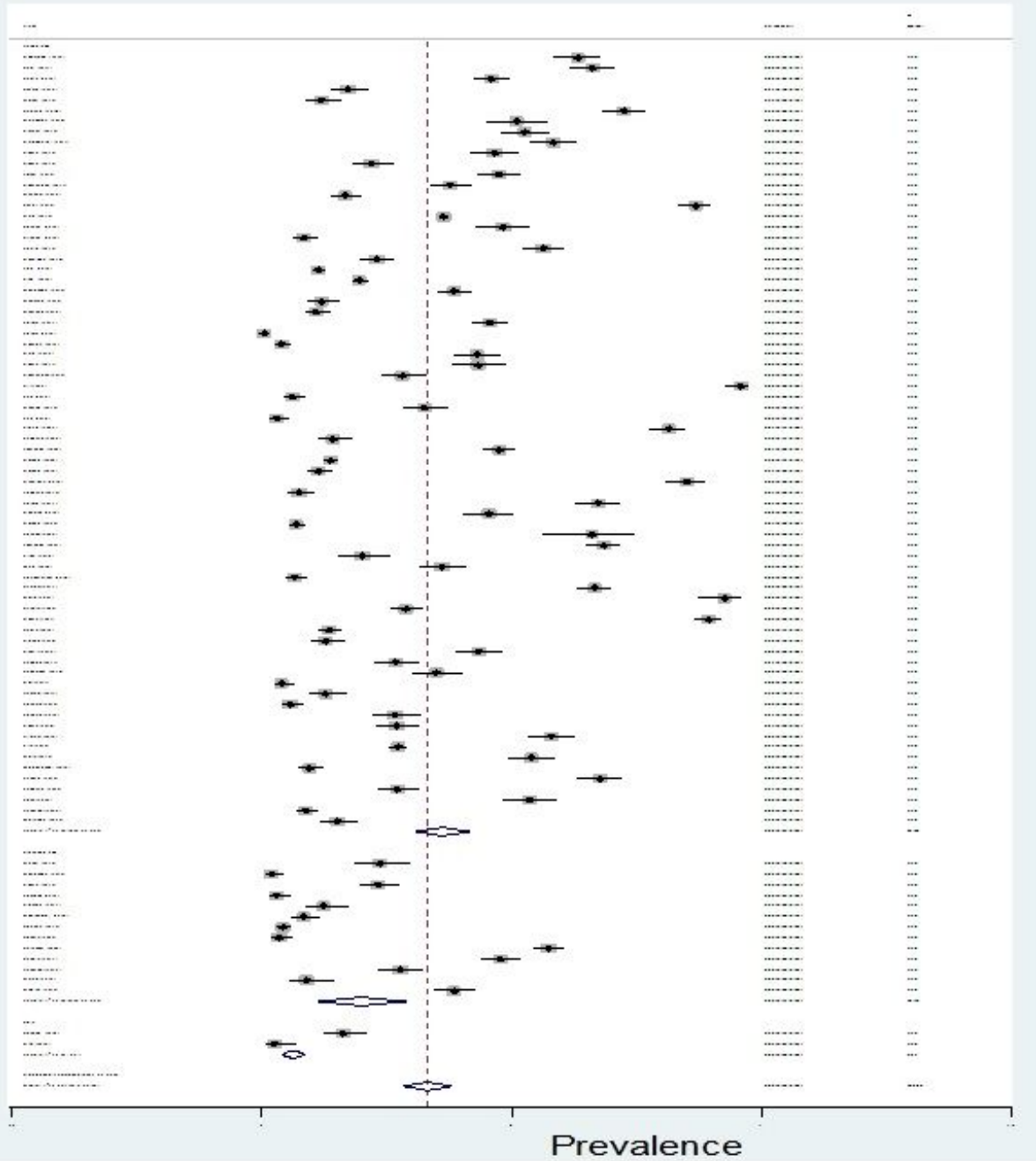

Figure 7

Forest plot showing prevalence of STHs by age 


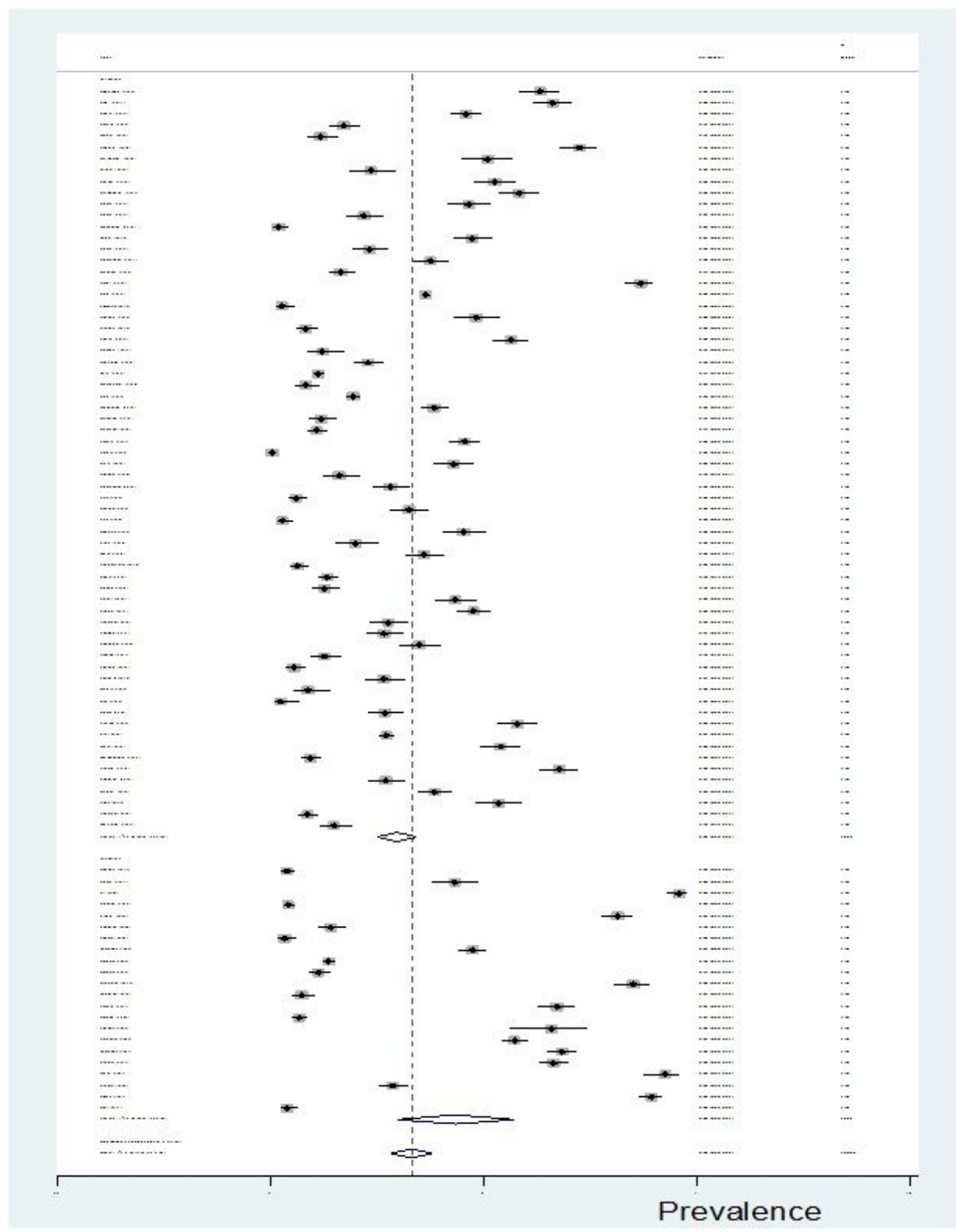

Figure 8

Forest plot showingprevalence of STHs by year of publication

\section{Supplementary Files}

This is a list of supplementary files associated with this preprint. Click to download.

- Supplementary1RegionaldistributionofA.docx 